\title{
A dislocation dynamics study of the strength of stacking fault tetrahedra. Part I: interactions with screw dislocations
}

\author{
E. Martinez , J. Marian , A. Arsenlis a , M. Victoria and J.M. Perlado \\ Lawrence Livermore National Laboratory, 7000 East Avenue, Livermore, CA 94551, USA; \\ Instituto de Fusión Nuclear, Universidad Politécnica de Madrid, Spain
}

\begin{abstract}
We present a comprehensive dislocation dynamics (DD) study of the strength of stacking fault tetrahedra (SFT) to screw dislocation glide in fec $\mathrm{Cu}$. Our methodology explicitly accounts for partial dislocation reactions in fec crystals, which allows us to provide more detailed insights into the dislocationSFT processes than previous DD studies. The resistance due to stacking fault surfaces to dislocation cutting has been computed using atomistic simulations and added in the form of a point stress to our DD methodology. We obtain a value of $1658.9 \mathrm{MPa}$, which translates into an extra force resolved on the glide plane that dislocations must overcome before they can penetrate SFTs. In fact, we see they do not, leading to two well differentiated regimes: (i) partial dislocation reactions, resulting in partial SFT damage, and (ii) impenetrable SFT resulting in the creation of Orowan loops. We obtain SFT strength maps as a function of dislocation glide plane-SFT intersection height, interaction orientation, and dislocation line length. In general SFTs are weaker obstacles the smaller the encountered triangular area is, which has allowed us to derive simple scaling laws with the slipped area as the only variable. These laws suffice to explain all strength curves and are used to derive a simple model of dislocation-SFT strength. The stresses required to break through obstacles in the 2.5-4.8-nm size range have been computed to be $100-300 \mathrm{MPa}$, in good agreement with some experimental estimations and molecular dynamics calculations.
\end{abstract}

Keywords: dislocation dynamics; stacking-fault tetrahedra; irradiation damage; Cu plasticity

\section{Introduction}

Energetic-particle irradiation of metallic materials results in microstructural changes that can lead to mechanical property degradation and failure In the low-to-intermediate temperature regime, these characteristic changes typically appear in the form of increases in the yield stress and a loss of ductility. The yield stress increase is attributed to the production of a high number density of radiation induced defects, which may include cavities, interstitial dislocation loops, stacking fault tetrahedra (SFT), etc. In face-centred cubic (fcc) metals, the most commonly observed irradiation induced defects are glissile interstitial loops, interstitial Frank loops, and SFTs [4]. Moreover, in low stacking-fault-energy metals, a large fraction $(\sim 25 \%$ in austenitic steels 
$40-90 \%$ in $\mathrm{Cu}[6-8]$ ) of the observed defects under neutron and ion irradiation are SFTs. In $\mathrm{Cu}$, SFTs typically appear in sizes of $2.5 \pm 0.5 \mathrm{~nm}$ and densities of the order of $10^{23} \mathrm{~m}^{-3}$ for experiments conducted at temperatures up to $\sim 250^{\circ} \mathrm{C} \quad$ For its part, the ductility loss is believed to be related to a reduction in strain hardening capability which, in turn, is caused by the softening effect due to dislocation channelling Indeed, transmission electron microscopy (TEM) observations of irradiated specimens prior to and following deformation reveal the formation of defect-free channels, which appear as clear, elongated bands, with very low visible defect densities

Prior to channelling, however, most experimental irradiation studies reveal homogeneous defect densities, with no appreciable spatial patterning This means that plastic localization along defect-free channels must be related to some SFT removal mechanism by dislocations originating at activated sources. Early models proposed that a single dislocation interacting with a radiation produced defect cluster led to sweeping or annihilation, producing decreased resistance for subsequent dislocation glide in a localized region of the material Dislocation-SFT interactions involve processes that occur on scales of the order of one nm or less, thus only accessible via state-of-the-art TEM techniques or computer simulations The majority of these latter works are molecular dynamics (MD) simulations of edge and screw dislocations interacting with small $(<5 \mathrm{~nm})$ tetrahedra. However, although these MD simulations provide detailed atomistic insights into the interaction mechanisms, the computational cost associated with large-scale simulations - needed to mitigate image and strain-rate artifacts - precludes its application to extract information of statistical nature such as strength laws (critical stresses), effects on plasticity (e.g. hardening), or other quantitative averages. In this sense, dislocation dynamics (DD) suggests itself as a natural approach for calculating all these magnitudes at relatively low computational cost while treating all dislocation interactions rigorously. The fundamental assumption to justify the use of DD is that interactions between SFTs and dislocations are governed by elasticity, which may break down when SFTs are very small and the interaction dynamics are dominated by short-range core effects. Several authors have indeed employed dislocation dynamics to study different aspects of irradiation-induced hardening in $\mathrm{Cu}$, but the particulars of the SFT removal mechanisms by dislocations are not considered explicitly due to the absence of partial dislocation reactions in their methodologies. Several analytical works have been published in an attempt to remove this limitation, where the elastic field between a screw dislocation and an SFT is solved explicitly The main conclusion extracted from these studies is that SFTs, even for small sizes, are very stable defects and do not budge under the influence of stress fields of the order of those produced by dislocations. However, the problem is that their analyses rely on rather rigid geometric assumptions and are quite limited in terms of the number of possible configurations that can be explored.

Recently, we have developed a dislocation dynamics methodology that allows for the explicit treatment of all partial dislocation reactions in fcc metals In this paper, we apply it to explore all the aforementioned issues, and to study and quantify the strength of SFTs to screw dislocation passage as a function of the reacting geometry and SFT size. In Section 2 we briefly describe our DD methodology; then, in Section 3, we present a validation exercise and results of SFT strength as a function of orientation for a 4.7-nm stacking fault tetrahedron when the intersection (glide) plane is varied from the SFT base to its apex. Also in Section 3 a line tension model is used to study the 
relationship between the SFT strength and its distance to the dislocation's end points. For the specific case of $70-\mathrm{nm}$ Frank-Read sources, we provide a model that relates the SFT strength to the area intersected by the dislocation's glide plane. In Section 4, we discuss the implications of this work and compare our simulations with MD simulations carried out under similar conditions. Finally, in Section 5 we provide the conclusions of our work.

\section{Methodology}

\subsection{Dislocation dynamics model}

Our methodology has been described in detail in [35], and is based on the non-singular, three-dimensional, discrete dislocation dynamics formulation implemented in the DDLab and ParaDis computer codes at Lawrence Livermore National Laboratory Our methods permit the treatment of all dislocation partials in fcc crystals explicitly, enabling us to directly simulate non-trivial characteristics of crystal plasticity in these materials, such as the formation of dislocation junctions, SFTs, cross-slip,

The key feature of the method is the introduction of a new vector $\gamma \mathbf{n}$, such that any dislocation segment acting as a stacking fault boundary has an additional force per unit length:

$$
\mathbf{f}_{\mathrm{SF}}=\xi \times \gamma \mathbf{n}
$$

where $\xi$ is a unit vector representing the dislocation line tangent, and $\gamma$ is the stacking fault energy. The $\mathbf{n}$ vectors, which need not be unitary in general, are different for each partial dislocation type, and are obtained from the known value of $\left\|\mathbf{f}_{\mathrm{SF}}\right\|=\gamma$ for Shockley partials, which gives $\mathbf{n}_{S p}=\langle(1 / \sqrt{3})(1 / \sqrt{3})(1 / \sqrt{3})\rangle$. In addition, the $\gamma \mathbf{n}$ ensemble must satisfy the following conservation rule at the nodal level:

$$
\sum_{i} \gamma \mathbf{n}_{i}=0
$$

where the summation runs over all the discrete dislocation segments converging on a given node. Equation 2 is in addition to the well-known Kirchoff-type rule for the continuity of the Burgers vector, $\sum_{i} \mathbf{b}_{i}=0$. As we have shown, these rules suffice to give the correct magnitude and direction of $\mathbf{f}_{\mathrm{SF}}$ for every partial dislocation segment in the simulations

Once all the forces acting upon a given segment are known, its velocity can be determined using the following kinetic law:

$$
\begin{aligned}
\mathbf{f} & =\mathcal{B} \mathbf{v} \\
\mathcal{B} & =B_{g}(\mathbf{m} \otimes \mathbf{m})+B_{c}(\mathbf{n} \otimes \mathbf{n})+B_{l}(\boldsymbol{\xi} \otimes \xi)
\end{aligned}
$$

where $\mathcal{B}$ is the drag coefficient tensor, $B_{g}$ and $B_{c}$ are temperature-dependent friction coefficients for motion along the glide and climb directions, and $B_{l}$ is the drag coefficient associated with a node in response to the configurational forces responsible for the equilibrium line shape acting on it Here, $\mathbf{n}$ and $\mathbf{m}$ are unit vectors defined as $\mathbf{n} \equiv(\mathbf{b} \times \xi) /\|\mathbf{b} \times \xi\|$ (note that, for Shockley partials, this definition of $\mathbf{n}$ coincides with that emanating from Equation 2), and $\mathbf{m} \equiv \mathbf{n} \times \xi$. This expression for $\mathcal{B}$ guarantees its 
invertibility so that the mobility tensor $\mathcal{M}=\mathcal{B}^{-1}$ can be obtained to calculate the velocities as: $\mathbf{v}=\mathcal{M} \mathbf{f}$. For this work, the critical coefficient is $B_{c} \gg B_{g}>B_{l}$, which is dislocation character dependent and constant in the low-velocity regime. The material parameters, including $B_{g}$, along with more details about the fitting procedure and the implementation, are all given in Ref. [35].

Our simulations are not influenced a priori by observations extracted from previous MD results. However, there is one physical aspect of the dislocation-SFT interaction process which appears naturally in MD and molecular statics (MS) but not in our simulations. This is the fact that a surface defect such as a stacking fault carries an associated stress field [37]. Unless they are amenable to representation by dislocation lines explicitly, these fields cannot be directly modelled using the current DD implementation, and no closed-form expressions exist for their evaluation. However, here we make use of a very advantageous property of grain boundary stress fields: they generally decay much faster than dislocation fields $(\sim \exp (-r)$ vs. $\sim 1 / r$ [38]). This allows us to simplify their treatment considerably by assuming that the surface can be modelled by a piecewise heaviside function that creates a local stress of zero range. Hence, to close our model, we need to calculate this critical stress, $\sigma_{\mathrm{SF}}$, required for a dislocation to traverse a stacking fault. This is done in Section 2.2, where $\sigma_{\mathrm{SF}}$ is computed using MS calculations and expressed in terms of an equivalent force.

\subsection{Calculation of the critical stress to traverse a stacking fault}

To calculate $\sigma_{\mathrm{SF}}$ we set up an fec crystallite containing 46080 atoms oriented along the [112]], [111], and [1ํㅣㄹ directions. We create an intrinsic stacking fault by removing one of the (111) planes, and an infinitely-straight screw BA dislocation dipole at a distance of $2.5 \mathrm{~nm}$ from the stacking fault. The screw dislocations dissociate on a [1-11] plane, i.e. forming $70.52^{\circ}$ with the stacking fault plane. The initial configuration can be seen in Figure 1(a), with the BA(c) dislocations and the $(d)$ stacking fault. We then apply shear stress to the computational box so as to make $(c)$ the plane of maximum resolved shear stress (RSS) while ensuring that no force is resolved on $(d)$. Our setup is similar to that

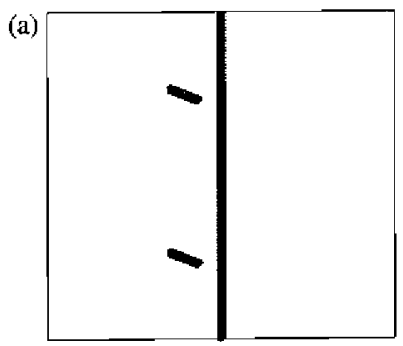

(b)

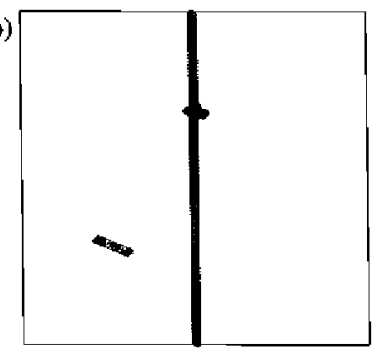

(c)

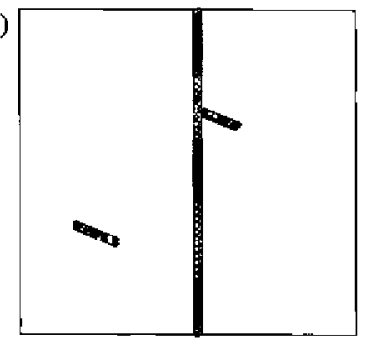

Figure 1. Atomistic configurations of a screw dislocation dipole in the presence of a stacking fault plane. Shear stress is applied on the dislocation glide plane but not on the fault plane. (a) Initial configuration visualized using the centrosymmetry deviation parameter. (b) The top dislocation pole is driven into the stacking fault, leading to its constriction at $1273.3 \mathrm{MPa}$. (c) At $\sigma_{\mathrm{SF}}=1658.9 \mathrm{MPa}$ the dislocation is able to traverse the stacking fault. 
used in other recent simulations As shown in Figure 1(b), the bottom dislocation pole glides away from the stacking fault, whereas the top one is driven into it. The latter proceeds towards the fault, but becomes arrested once it reaches it. Upon increasing the RSS, the dislocation starts constricting until, at $1273.3 \mathrm{MPa}$, full constriction is attained (see cross-like structure in Figure 1(b)), and becomes pinned at the fault. From here, only after the RSS is increased to a value of $\sigma_{\mathrm{SF}}=1658.9 \mathrm{MPa}$ is the dislocation able to penetrate the stacking fault and move on the other side of the crystal (Figure 1(c)). This result suffers from the limitations associated with finite-size atomistic calculations, such as periodic boundary images, dipole self-interactions, interatomic potential approximations, etc.

It is worth noting that $\sigma_{\mathrm{SF}}$ is independent of the sign of the applied stress, i.e. the results are the same regardless of which pole is driven into the stacking fault. The force per unit length resolved on the $(b)$ plane on a screw dislocation due to $\sigma_{\mathrm{SF}}$ is $f^{*}=9.58 \times 10^{-11}$ $\mathrm{N} b^{-1}$, where $b$ is the modulus of the perfect Burgers vector. Our method to incorporate this information into our model is as follows. First, we detect when a node has reached a stacking fault; then, we compute its local force during each time-step; if the projection of the nodal force in the direction orthogonal to the stacking fault surface is greater than the prescribed value given by $f^{*}$, the node penetrates the fault; else, the node remains at the stacking fault surface.

In addition, supplementary MS simulations taking the constricted state in Figure 1(b) as the starting configuration have been carried out with stress also applied on the fault plane. In such conditions, dislocations are seen to dissociate on the fault plane (cross-slip), partially removing it by cancellation with their own intrinsic fault. Thus, cross-slip is indeed an accessible transition for the constricted dislocation, especially in the presence of the complex tetrahedron geometry and associated stress fields.

\subsection{Initial geometry and boundary conditions}

All the simulations presented in this work consist of finite dislocation segments - ranging 70 to $150 \mathrm{~nm}$ in length - pinned at their end points, i.e. akin to simulating a Frank-Read source operating on a single slip plane. The character of the dislocation is assigned at the beginning of the simulation, when the dislocation line coincides with a straight segment joining both end points. The SFT is initially placed at a distance of one or more lattice parameters from the dislocation line.

Keeping these constraints in mind, as a preliminary check, we have performed a simple DD simulation whereby we apply shear stress incrementally to drive a dissociated screw dislocation into an 4.7-nm SFT, as shown in Figure 2a. When the dislocation nodes of the leading Shockley partial come into contact with the stacking-fault surface they become pinned (Figure 2b), corresponding to the repulsion of magnitude $f^{*}$ exerted by the facet plus the effect of the elastic fields stemming from the SFT stair-rods (not present in Figure 1). This repulsion due to the tetrahedron is greater than the force required for dislocation constriction, so that when the stress is increased the trailing partial eventually reacts with the pinned leading partial. This leads to full constriction across the SFT facet (Figure 2c) at an applied stress of approximately $145 \mathrm{MPa}$. As we shall see, this constricted configuration, locked at the stacking-fault plane of the SFT can result in several reaction pathways depending on the reacting geometry and stress conditions. 
(a)

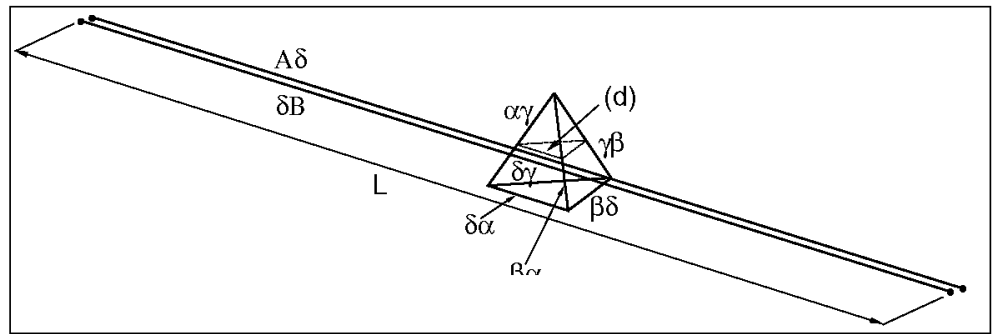

(b)

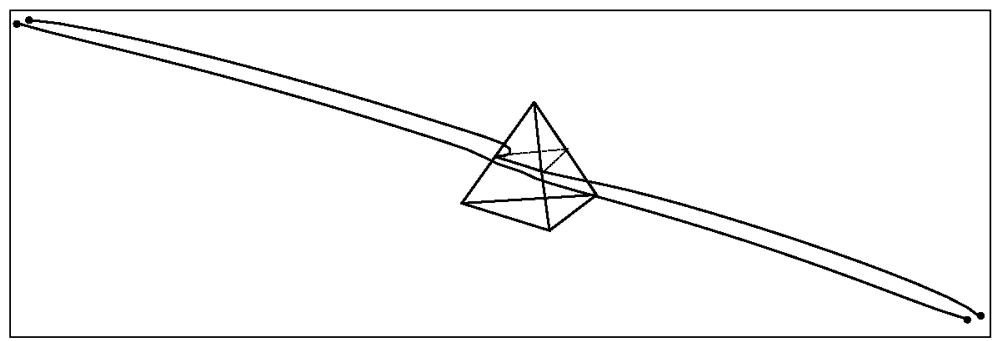

(c)

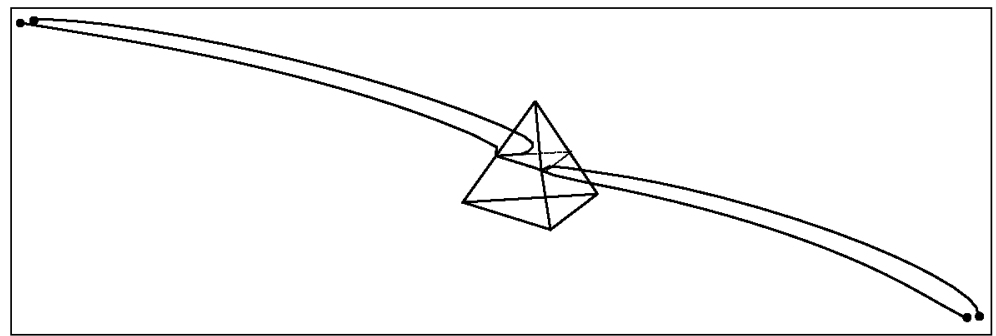

Figure 2. Sequence of snapshots from a DD simulation of a dissociated screw dislocation being driven toward an SFT at its mid-height plane face-on. (a) Initial geometry including the tetrahedron orientation, the dislocation splitting and the area of the SFT intersected by the glide plane (labelled '(d)'). $L$ is the separation between the pinning points of the Frank-Read source. (b) Constriction starts at the edge of the facet, in contact with one of the stair-rod dislocations. (c) Full constriction is attained before $f^{*}$ is surpassed. Dislocation segments are coloured according to their Burgers vector: Shockley partials in blue, stair-rods in green, and perfect dislocations in red.

Unless otherwise noted, the configuration shown in Figure 2(a) will be taken as the starting geometry for all the simulations performed in this work.

\section{Results}

The simplified model presented in Section 2 suffices to provide a satisfactory framework to study dislocation interactions with SFTs. In this section we first perform a simple exercise of validation against specifically-tailored atomistic simulations of SFT morphology and stability as a function of size. Then, we study the interaction mechanism between screw dislocations and stacking-fault tetrahedra for several reacting geometries. Finally, we provide a detailed study of SFT strength as a function of the distance of the glide plane from the SFT base as a function of tetrahedron size. 


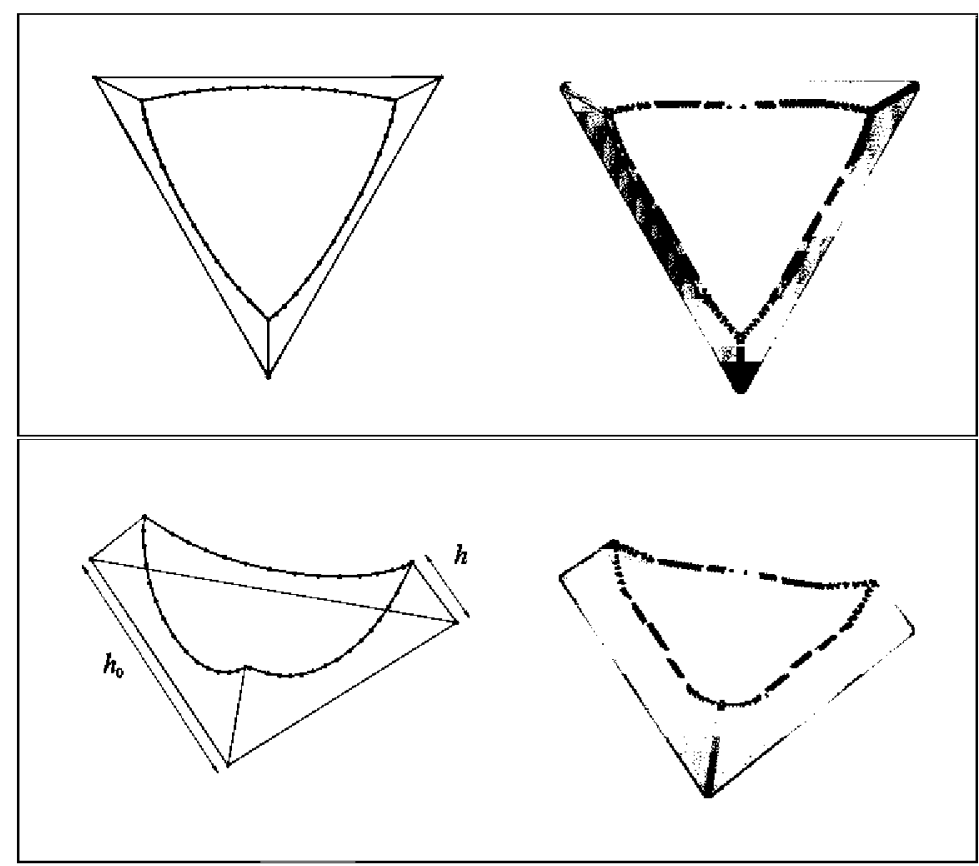

Figure 3. Two different views of a 25.6-nm truncated SFT as obtained with DD. Dislocation segments are colour-coded according to their Burgers vector: blue represents Shockley partials, and green stair-rod dislocations. Two MD snapshots for the same size SFT are shown for comparison, with very good agreement.

\subsection{Formation and stability of stacking fault tetrahedra}

The formation of stacking fault tetrahedra from vacancy platelets in quenched metals and from the collapse of irradiation cascades has been studied in detail, both experimentally and using simulations In standard dislocation theory, the formation of stacking fault tetrahedra from dissociated Frank loops is governed by two competing elastic processes, namely, the reactions of pairs of Shockley partials into stair rod dislocations along the SFT edges, and the creation of stacking faults on each of the facets of the $\{111\}$ tetrahedron

According to this mechanism, known as Silcox-Hirsch [49], the energetic balance between these two processes determines the equilibrium SFT structure. In a recent publication, we have demonstrated that this mechanism is recovered for $<22$-nm Frank loops in $\mathrm{Cu}$ using our DD methodology However, for larger sizes, the elastic energy reduction due to reactions of the type $(1 / 6)+(1 / 6) \rightarrow(1 / 6) \quad$ along the tetrahedron edges may not be sufficient to sustain the increase in stacking fault area, which leads to equilibrium configurations known as truncated SFTs.

Here we use DD simulations of triangular Frank loops in the $0-108.5-\mathrm{nm}$ size range to study the morphology of the resulting SFTs and establish the critical truncation size. For the purpose of validation, we have performed a number of molecular dynamics simulations for tetrahedron sizes of 4.1, 19.9, 22.5 and $25.6 \mathrm{~nm}$. In Figure 3 we compare the morphology of the 25.6-nm SFT, obtained with dislocation dynamics, with the final 


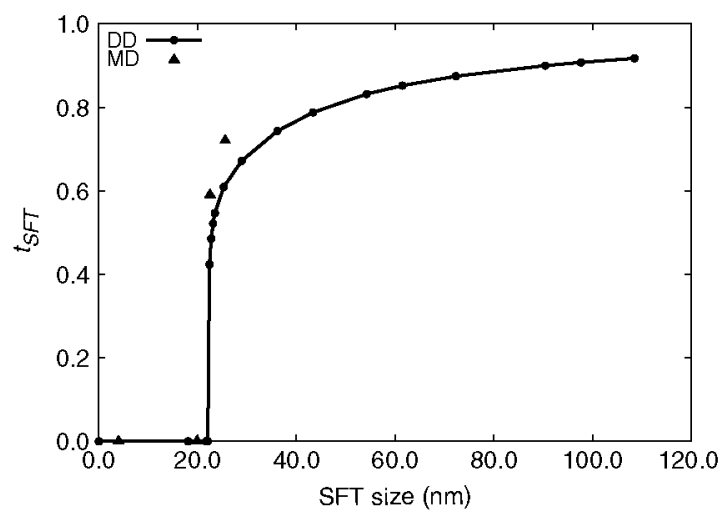

Figure 4. Truncation factor, $t_{\mathrm{SFT}}$, for stacking fault tetrahedra as function of their size. $t_{\mathrm{SFT}}$ is zero, indicating perfect SFTs, up to a size of $22 \mathrm{~nm}$, which can be considered the size limit for full SFT stability in $\mathrm{Cu}$. After that, the truncation factor grows abruptly to values of 0.75 at $\sim 40 \mathrm{~nm}$, after which it is seen to vary smoothly up to the largest simulated SFT size of $108.5 \mathrm{~nm}$ (Note: a value of $t_{\mathrm{SFT}}=1$ indicates a non-dissociated ('flat') triangular Frank loop). Four MD points, at 4.1, 19.9, 22.5 , and $25.6 \mathrm{~nm}$, are included for comparison.

structure obtained from atomistic relaxations performed for a triangular platelet of the same size containing 5058 vacancies. The figure clearly shows the good agreement between the DD and MD results.

In Figure 4 we plot the truncation factor, defined as $t_{\mathrm{SFT}}=1-h / h_{0}$, as a function of the length $h_{0}$ of the initial triangular Frank loop. $h$ is the characteristic length of the truncated dimension (see Figure 3). A value of $t_{\mathrm{SFT}}=0$ represents a perfect SFT, whereas a value of $t_{\mathrm{SFT}}=1$ indicates a non-dissociated Frank loop. From the figure, the maximum size for a perfect SFT in $\mathrm{Cu}$ is $22.0 \mathrm{~nm}$. Beyond this value, the $t_{\mathrm{SFT}}$ is seen to grow abruptly, e.g. $60 \%$ for $h_{0}=25.3 \mathrm{~nm}$, before gradually levelling off into a monotonic function that approaches unity asymptotically. For the largest simulated SFT size of $108.5 \mathrm{~nm}$, we obtained a truncation factor of $91.7 \%$. Note that a $108.5-\mathrm{nm}$ SFT corresponds to a triangular platelet containing 64375 vacancies, something for which no MD simulation exists. Two of the MD data points provided for comparison, 4.1 and $19.9 \mathrm{~nm}$, match the DD results and fall below the truncation threshold of $h<22.0 \mathrm{~nm}$. For the 22.5 and 25.6-nm SFTs, both methods give truncated tetrahedra, although, while the agreement between DD and MD for the former structure is excellent, in the latter case there is an $\sim 18 \%$ disparity in the calculated truncation factor. This can be rationalized in terms of the different curvatures observed in Figure 3, which lead to different equilibrium values of $h$. Second-order effects, such as non-linear and/or anisotropic elasticity, core-core interactions, and periodic image effects, all of them present in the MD simulations, may also contribute to this discrepancy, although, notably, the stacking-fault areas enclosed by each SFT facet are very close in both cases.

\subsection{Interaction mechanisms between screw dislocations and SFTs}

In the classical picture of Kimura and Maddin screw dislocations are more effective at annihilating SFTs due to their ability to cross-slip and remove the stacking faults on each 
of the tetrahedron's facets and, more recently,

Indeed, early TEM observations by Johnson and Hirsch show that screw dislocations are more efficient than edge dislocations at removing radiation obstacles. Several recent $\mathrm{MD}$ studies are in qualitative agreement with this observation However, these atomistic works differ on the SFT absorption mechanisms, ranging from multiple shearing, absorption of vacancies into super-jogs and spirals, or transformation of the defects into others with reduced resistance. In this section we present results of DD simulations of SFT-screw dislocation interactions in fec $\mathrm{Cu}$. We study four different geometries, namely, face-on and edge-on interactions between a 70 -nm-long $\pm(1 / 2)[1 \overline{1} 0](111)(\mathrm{AB} / \mathrm{BA}(d))$ screw dislocation, and, for comparison with the MD simulations a 4.7-nm SFT. The simulations are performed with stress resolved only on the glide plane, with dislocation velocities in the $0.1-10-\mathrm{m} \mathrm{s}^{-1}$ range. All simulations are stress-driven. For simplicity, Thompson's notation is used to describe all dislocation reactions.

\subsubsection{Face-on interaction mechanism}

We apply shear stress incrementally on plane $(d)$ to drive a screw dislocation towards a stacking-fault tetrahedron. For the face-on case, we immediately reach the state shown in Figure $2 \mathrm{c}$, i.e. when the leading $\triangle \delta$ Shockley partial reaches the $(c)$ facet, it stops $\left(f_{i}<f^{*}\right.$, where $i$ is the segment index), resulting in a constricted AB dislocation by collapse of the trailing partial. Following constriction, the perfect $\mathrm{AB}$ dislocation now dissociates on the (c) plane into $\mathrm{A} \gamma$ and $\gamma \mathrm{B}$. This results in the removal of the stacking fault area enclosed by the $\mathrm{A} \gamma$ and $\gamma \mathrm{B}$ partials. This process, illustrated in Figure 5(1), is common to all interaction 'heights' but we have seen that, from that point on, the mechanism proceeds differently depending on the distance from the base. For distances $>0.23 h_{0}$, the basal stair-rod $-\delta \gamma$ in this case - is able to exert a sufficiently strong attraction on the lower partial and trigger a string of reactions, sequentially shown in Figure 5, which we proceed to describe in detail. In the following, there is a direct correspondence among the frames shown in the figure and the bullets describing the mechanism:

(1) As just mentioned, the constricted perfect screw dislocation $\mathrm{AB}$ dissociates on the SFTs (c) facet as an A $\gamma$ and a $\gamma \mathrm{B}$ Shockley pair. This dissociation now produces a stacking fault of opposite sign to the existing one, resulting in the 'healing' of the $(11 \overline{1})$ facet plane. Thus, the reactions on $(c)$ are:

$$
\mathrm{AB} \rightarrow \mathrm{A} \gamma+\gamma \mathrm{B}
$$

(2) Pulled by the basal $\delta \gamma$ dislocation, the $\gamma$ B Shockley quickly moves down facet (c), removing the stacking fault in its wake. When it reaches the base, it reacts with $\delta \gamma$ :

$$
\gamma \mathrm{B}+\delta \gamma \rightarrow \delta \mathrm{B}
$$

In addition, when it reaches the facet's edge, it reacts with $\alpha \gamma$ :

$$
\gamma \mathrm{B}+\alpha \gamma \rightarrow \alpha \mathrm{B}
$$

(3) The $\alpha \mathrm{B}$ and $\delta \mathrm{B}$ Shockleys are glissile on $(a)$ and $(d)$ respectively, which they proceed to sweep. 

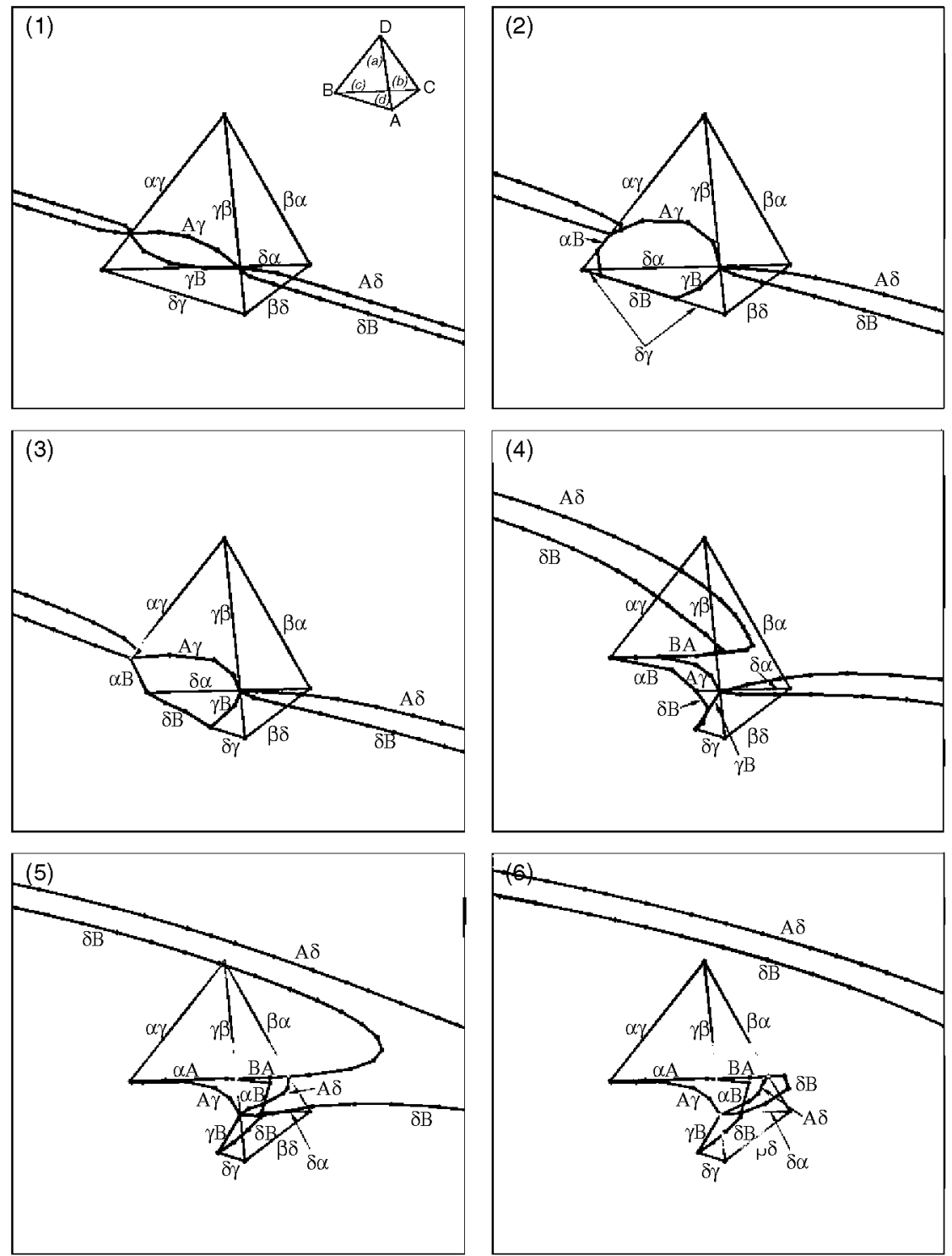

Figure 5. Sequence of snapshots from a DD simulation illustrating the interaction mechanism between a screw dislocation and a 4.7-nm SFT face-on. Segments are colour-coded according to their Burgers vector: Shockley partials are blue, stair-rod dislocations are green, Frank partials black, and perfect dislocations are shown in red. The inset in frame (1) shows the orientation of the Thompson tetrahedron used for this set of simulations. For colour, see online.

(4) The left outer arm of the original dissociated AB dislocation curves around the SFT, collapsing onto itself on face $(a)$ as a perfect BA dislocation segment. ${ }^{1}$ It is worth mentioning that, at this stage, Osetsky et al. see an alternative mechanism using MD by which the $\gamma \mathrm{B}$ partial (curved until it reverses sign into $\mathrm{B} \gamma$ ) 
collapses onto the $\gamma \beta$ stair-rod, giving rise to a high-energy $\mathrm{B} \beta$ dislocation This Frank segment is unstable and quickly dissociates, emitting a perfect dislocation node (e.g. BA or BD) and leaving a Shockley partial segment behind $(\mathrm{A} \beta$ or $\mathrm{D} \beta)$. The new perfect dislocation may dissociate on $(c)$ or $(b)$ forming a super-jog. In principle, this mechanism is completely plausible within our methodology, although it does not occur in our case due to the local stress conditions, which prevent the constriction between $\mathrm{B} \gamma$ and $\gamma \beta$.

(5) The constricted BA segment reacts with the nearby $\alpha \mathrm{B}$ Shockley, giving rise to a $\alpha$ A Frank segment.

Reactions on $(a)$ :

$$
\mathrm{BA}+\alpha \mathrm{B} \rightarrow \alpha \mathrm{A}
$$

At the same time, after curving around the defect, the leading partial reconnects with itself, detaching.

(6) The combination of applied stress and $f_{\mathrm{SF}}$ pull originating from the detached leading partial results in the reconnection of the trailing partial, downstream of the SFT, freeing the entire screw dislocation from the defect. The detachment of the $\delta \mathrm{B}$ Shockley leaves a ledge, which protrudes from the $(b)$ facet and remains as a dissociated $\mathrm{AB}$ segment. The structure and evolution of this ledge might be different if we had considered finite displacements, i.e. accounting for the shear (of magnitude $b_{\mathrm{Sp}}$ ) transported by the dislocation (our simulations do not). In fact, we speculate that this could trigger the dissociation of $\alpha \mathrm{A}$ into $\alpha \delta$ and $\delta \mathrm{A}$. The $\delta \mathrm{A}$ Shockley could in turn sweep the glide plane back into the existing $\mathrm{A} \gamma$ to give the remaining stair-rod to convert the leftover configuration of Figure 5(6) into a smaller SFT with base on the original glide plane.

It is worth emphasizing that, because shear stress is only applied on the glide plane, all processes not occurring on $(d)$-type planes are driven solely by spontaneous dislocation reactions. As we have pointed out, the spontaneous reactions occurring on plane $(c)$ are suppressed beyond a critical distance between the initial glide plane and the SFT base. In such cases, the attractive force between the stair-rod and the Shockley partial dislocations on $(c)$ is not sufficient to pull the two segments together and the SFT behaves as an impenetrable obstacle, leaving an Orowan loop behind (cf. Figure 6). For the 4.7-nm $\mathrm{Cu}$ SFT, this critical height lies between $0.23 h_{0}$ and $0.31 h_{0}$, although this value can be controlled by applying stress on the facet plane. Indeed, we have seen that for an RSS of $\approx 20 \mathrm{MPa}$ on $(c)$, the critical height ascends to approximately $0.60 h_{0}$. For the BA dislocation, the mechanisms observed are simply a mirror image of those just described and, therefore, this interaction is qualitatively independent of the Burgers vector orientation (although the stresses may differ, as we shall see in Section 3.3).

Interestingly, under no circumstances was the dislocation seen to be able to overcome the repulsive force due to the stacking fault, $f^{*}$, which would lead to shearing of the SFT. Thus, we establish two interaction regimes, separated by a critical distance from the tetrahedron's base: (i) reaction-induced partial SFT damage below it, and, (ii), Orowan looping above it. These mechanisms are in excellent agreement with the MD results by Lee et al. [28] and, to a lesser degree, Osetsky experimental observations by Robach Together with these works and recent our results provide sufficient new mechanistic information to effectively supersede Kimura and Maddin's original 


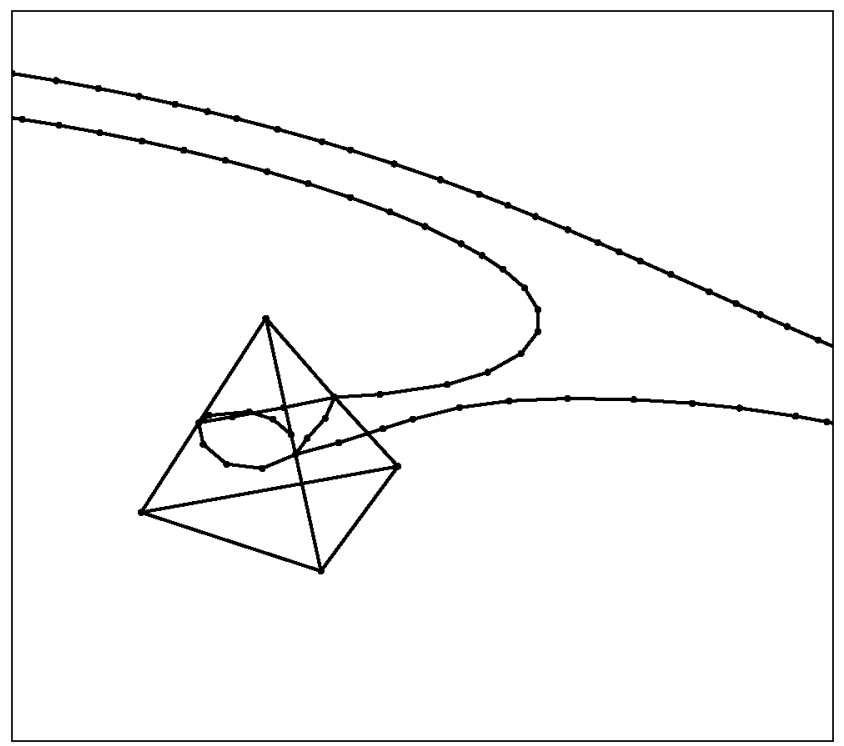

Figure 6. Mechanism of Orowan looping around an SFT as obtained with our DD simulations. The trailing dislocation is just about to detach, leaving a triangular dislocation loop (in red: perfect Burgers vector) behind. Upon relaxation, each segment of the loop may dissociate either on the facet plane or on the glide plane, depending on the dislocation character relative to the facet orientation. For colour, see online.

mechanism of SFT absorption Furthermore, our results support the assumption that an isotropic elastic treatment suffices to describe the interaction between a screw dislocation and a stacking-fault tetrahedron, at least in this SFT size range. This certainty enables us to now apply the method to more quantitative aspects of the effect of irradiation on fcc crystal plasticity.

In Figure 7 we show the stress displacement curve for the mechanisms shown in Figures 5 and 6 . Numbered frames in Figure 5 have a direct correspondence with the numbered labels on the stress-displacement curve for $h=0.23 h_{0}$. Horizontal steps indicate rapid dislocation glide, both on the glide plane - e.g. (5), release of the leading partial, and (6), release of the trailing partial - as well as on the SFT facets - such as (1), dissociation of the original dislocation on the $(d)$ facet plane. These horizontal steps punctuate regions of rapid stress buildup.

\subsubsection{Edge-on interaction mechanism}

The starting geometry is again an $\mathrm{AB}(d)$ perfect dislocation dissociated into two $\delta \mathrm{B}$ (leading) and $\mathrm{A} \delta$ (trailing) Shockley partials on a glide plane situated at a distance $0.23 h_{0}$ above the SFT base. The mechanism is described in the following, with direct correspondence among the different steps and the snapshots shown in Figure 8:

(1) Upon contact with the edge, the leading partial hugs the tetrahedron and forms a 'V'-shaped segment on the facets $(a)$ and $(b)$ of the tetrahedron. This stagnates the process until the trailing partial catches up and produces two locally constricted 


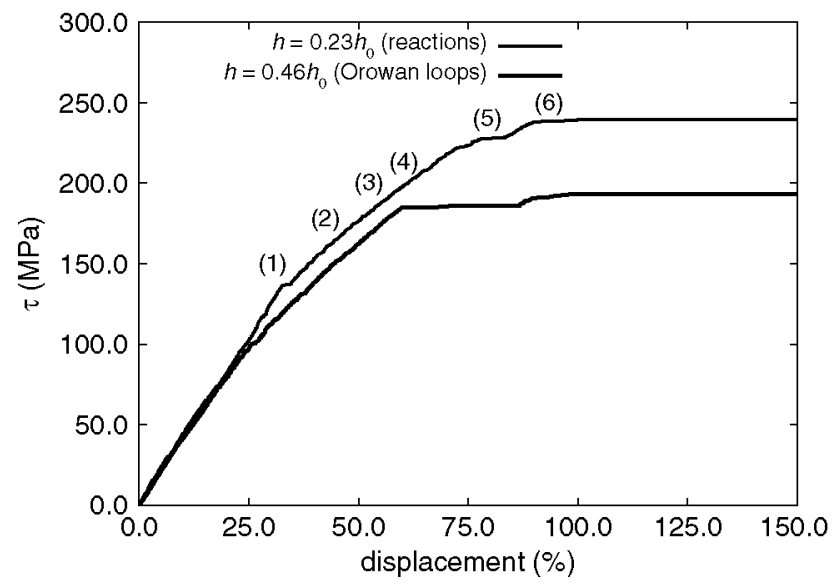

Figure 7. Stress-displacement curves for the two regimes occurring for the face-on interaction. The blue (upper) curve corresponds to the interaction shown in figure 5, where the different snapshots have been labelled correspondingly. The red (lower) curve is representative of heights where the operating mechanism is Orowan looping. The displacement is measured on the glide plane and varies from the initial dislocation-SFT point of contact $(0 \%)$ to the SFT exit point $(100 \%)$ plus a certain amount of glide downstream of the defect.

segments on the said facets. These constricted segments are now perfect $60^{\circ}$ mixed $\mathrm{AB}$ dislocations, and, as such, can only dissociate into a sessile $\mathrm{A} \alpha$ Frank segment and a $\alpha \mathrm{B}$ Shockley partial on the (a) plane, and into a $\mathrm{A} \beta$ and $\beta \mathrm{B}$ on $(b)$. These two dissociations, however, have different effects depending on what facet they occur. For example, on plane $(b)$, the basal stair-rod dislocation $\beta \delta$ attracts the A $\beta$ Shockley, which sweeps the stacking fault on its way, leaving a perfect crystal behind. Conversely, on plane (a), the $\delta \alpha$ stair-rod repels the $\alpha \mathrm{B}$ Shockley, making the stress-assisted sweep much more difficult.

Reactions on $(a)$ :

$$
\mathrm{AB} \rightarrow \mathrm{A} \alpha+\alpha \mathrm{B}
$$

Reactions on $(b)$ :

$$
\mathrm{AB} \rightarrow \beta \mathrm{B}+\mathrm{A} \beta
$$

As for the face-on case, due to the local constriction states that occur on the SFT facets, this mechanism is independent of the Burgers vector sign ( $\mathrm{AB}$ vs. BA), and the equivalent mechanisms are just mirror images of the one described here.

(2) After sweeping the portion of the $(b)$ facet below the glide plane, the $\mathrm{A} \beta$ Shockley reacts with $\beta \delta$ into an A $\delta$ Shockley partial on the SFT base. This partial is of course glissile on the base, whose stacking fault is removed almost completely. Reactions on $(d)$ :

$$
\mathrm{A} \beta+\beta \delta \rightarrow \mathrm{A} \delta
$$



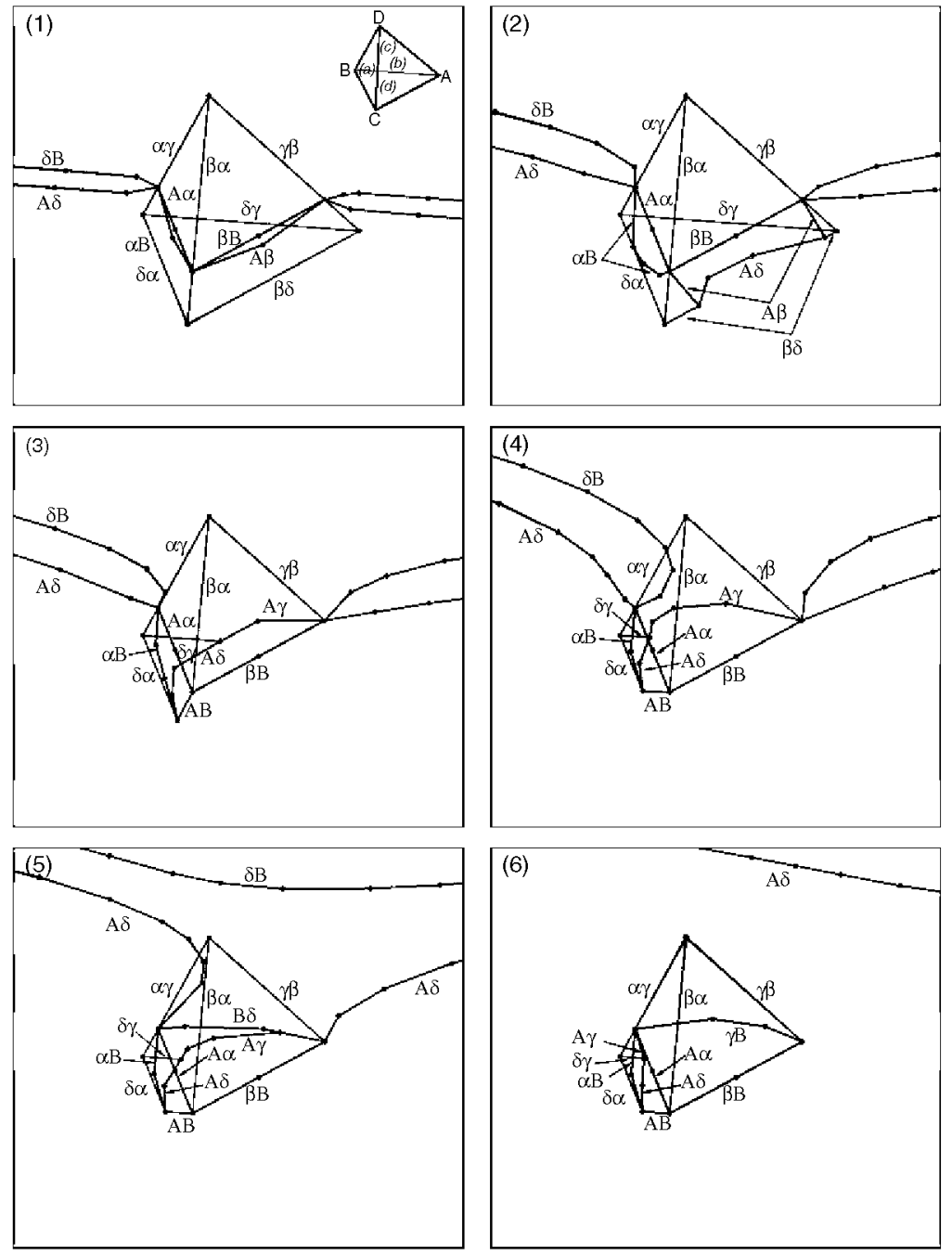

Figure 8. Sequence of snapshots from a DD simulation illustrating the interaction mechanism between a screw dislocation and a 4.6-nm SFT edge-on. Segments are colour-coded according to their Burgers vector: Shockley partials are blue, stair-rod dislocations are green, Frank partials black, and perfect dislocations are shown in red. The inset in frame (1) shows the orientation of the Thompson tetrahedron used for this set of simulations.

(3) As it proceeds through the basal plane, the A $\delta$ Shockley partial reacts with the $\delta \gamma$ stair-rod acting as the lower limit of the SFTs back face $(c)$, giving rise to a A $\gamma$ dislocation. This dislocation removes the stacking fault on (c) from the base up to the glide plane.

Reactions on $(c)$ :

$$
\mathrm{A} \delta+\delta \gamma \rightarrow \mathrm{A} \gamma
$$


On the original impinging edge of the SFT (defined by the $\beta \alpha$ stair-rod), below the glide plane, the original $A \beta$ Shockley on $(b)$ reacts with $\beta \alpha$ on its way down the face and, in turn, with the other Shockley partial that remains in $(a), \alpha \mathrm{B}$, to give the original perfect dislocation $\mathrm{B}$ :

$$
\mathrm{A} \beta+\beta \alpha+\alpha \mathrm{B} \rightarrow \mathrm{AB}
$$

(4) Under the influence of the applied stress, partials $\delta \mathrm{B}$ and $\mathrm{A} \delta$ curve around the tetrahedron. The base node of the $\mathrm{AB}$ segment is dragged along the lower edge of the $(a)$ facet, decreasing the length of the original $\delta \alpha$ stair-rod dislocation.

(5) In an 'Orowan'-type process, the leading partial $\delta \mathrm{B}$ reconnects with itself after significant curvature, and leaves a residual $\mathrm{B} \delta$ (the line tangent changes sign) segment on the backside (c) of the tetrahedron (on the glide plane). The detachment of the leading partial accelerates the reconnection of the trailing partial.

(6) The collapse of the trailing partial, as $\delta \mathrm{A}$ (see endnote 1) upon facet (c) causes the following reaction:

$$
\delta \mathrm{A}+\mathrm{A} \gamma+\mathrm{B} \delta \rightarrow \gamma \mathrm{B}
$$

and the resulting structure consists of an SFT whose portion from the glide plane down to the base has essentially been removed. Conceivably, all Frank partials left on the glide plane could in principle dissociate to close in a new base at the glide plane level, giving rise to a smaller, closed SFT

As for the face-on case, steps (1)-(6) give the operating mechanism up to the third height tried $\left(h=0.23 h_{0}\right)$. At the point immediately above $\left(h=0.31 h_{0}\right)$, the observed mechanism is Orowan looping all the way up to the SFT apex. We emphasize that the critical transition height can be increased and controlled by applying stress on the facet planes.

\subsubsection{Basal mechanisms}

In this case, the mechanisms for the $\mathrm{AB}$ and $\mathrm{BA}$ perfect dislocations are different, as, instead of pinning leading to constriction, the Shockley partials can react freely with the basal stair-rods. For clarity, we describe the interaction mechanisms in detail for the face-on and edge-on cases in Appendices A and B respectively. The main observations are summarized here:

- Reaction AB face-on (Appendix A.1): the base is removed and the SFT is partially absorbed.

- Reaction BA face-on (Appendix A.2): the dislocation curves around the base, leaving an Orowan loop.

- Reaction AB edge-on (Appendix B.1): the SFT is left intact.

- Reaction BA edge-on (Appendix B.2): the base is removed and the SFT is partially absorbed. 


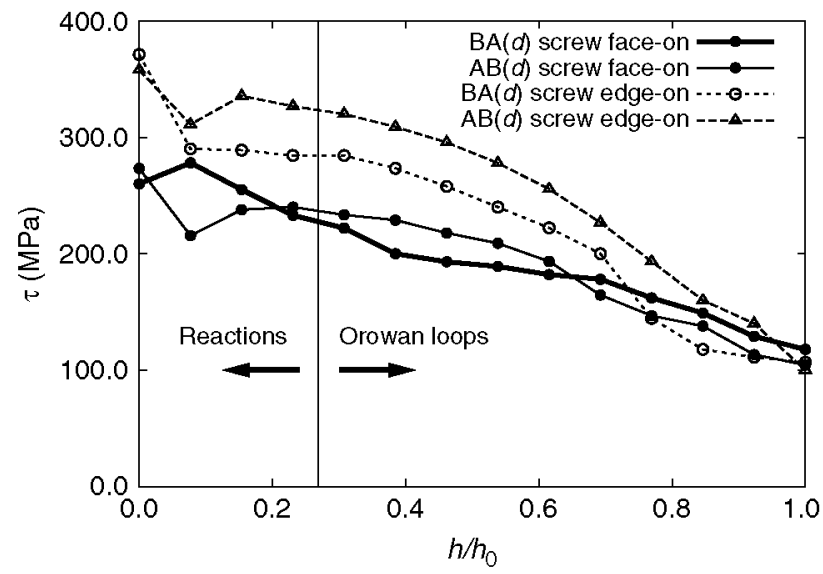

Figure 9. SFT strength as a function of the normalized interaction height, $h / h_{0}$, for a screw dislocation impinging on a $h_{0}=4.7-\mathrm{nm}$ SFT face and edge-on. A value of $h / h_{0}=0$ corresponds to the SFT base. Both directions of the Burgers vectors are considered, with a noticeable effect. The vertical line marks the transition from a reaction-based mechanism to an Orowan loop mechanism.

\subsection{Strength of SFTs to screw dislocation passage}

In order to study the SFT strength as a function of the distance between the dislocation glide plane and the SFT base, we have extended the above simulations to the entire range of 'heights' going from the SFT base all the way up to its apex. The simulations are stressdriven, with shear stress applied only on the glide plane until the dislocation is able to traverse the obstacle. In total, 14 different heights were explored in each case. Figure 9 shows strength results for a $h_{0}=4.7-\mathrm{nm}$ perfect SFT as a function of the normalized height, $h / h_{0}$. In all cases the reaction mechanisms are those described, respectively, in Sections 3.2.1 and 3.2.2, except at the bases $\left(h / h_{0}=0.0\right)$, which are given in Section 3.2.3 and Appendices $\mathrm{A}$ and $\mathrm{B}$.

In general, the SFT becomes progressively weaker with distance from the base, the exception being at or close to the base itself, where, as we have seen, the interaction mechanism differs from that observed elsewhere. For example, for the $\mathrm{BA}(d)$ dislocation impinging face-on, the basal stress $\tau_{b}$ is slightly lower than the first point tried, just $0.35 \mathrm{~nm}$ above the base. Conversely, for all the other cases, $\tau_{b}$ is significantly higher than the stresses calculated at higher intersection locations, which leads to appreciable softening already at the first point considered. Above $h \approx 0.27 h_{0}$, Orowan's mechanism sets in for both face-on and edge-on interactions, resulting in the monotonically decreasing behaviour observed in Figure 9. No shearing ${ }^{1}$ was observed for any of the cases considered here. Generally speaking, more stress is required to traverse the SFT edge-on than face-on. However, at the apex the dislocation is able to traverse the SFT more easily when it is oriented edge-on - 118 vs. $100 \mathrm{MPa}$ (collision stresses). This is presumably related to the coupling between the stress fields of the dislocation and the tetrahedron, as no reactions among dislocations occur at this location. It is worth remarking that despite the absence of reactions, we treat the SFT apex as a dislocation point, thus offering resistance to dislocation passage. Turning off the collisions feature of our codes 


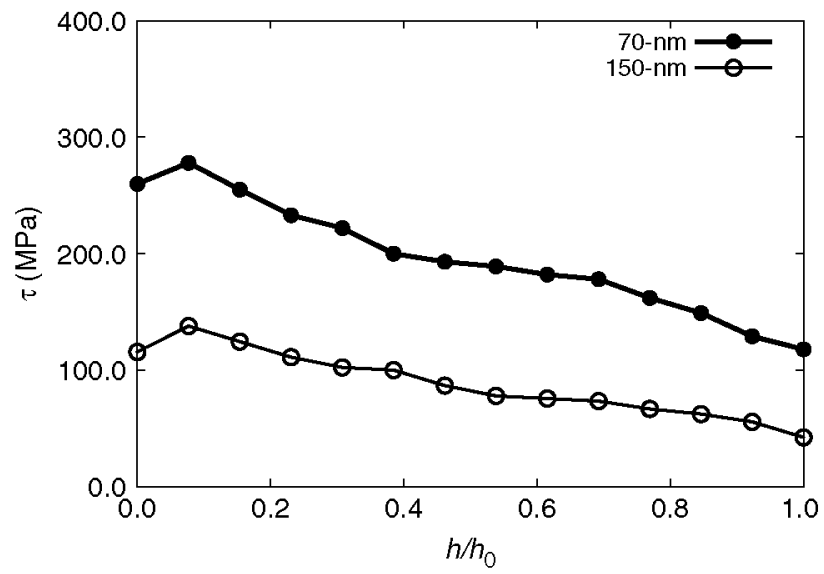

Figure 10. SFT strength to a BA $(d)$ screw dislocation impinging face-on for initial dislocation legths of 70 and $150 \mathrm{~nm}$.

for this point, the measured stress is much lower, 86.7 and 42.2 MPa for the face-on and edge-on cases respectively. It is reasonable to expect that the real value will lie in between these two results, which essentially act as upper and lower-bound estimates of the apex strength.

Another important aspect to keep in mind is the effect of the dislocation segment length, $L$, on the results presented in this paper. Our simulations have been carried out with 70-nm dislocations pinned at the end points, which corresponds to a forest dislocation (or obstacle) density of $\rho_{f} \approx L^{-2}=2.04 \times 10^{10} \mathrm{~cm}^{-2}$. The separation between pinning points also gives the curvature under stress of the dislocation, as dictated by the accumulated line tension $\sigma_{l}=\mu b / L$. Preliminary calculations using $150-\mathrm{nm}$ segments indicate that the effect is notable: the SFT strength for $150-\mathrm{nm}$ dislocations is approximately $42 \%$ of that for $70-\mathrm{nm}$ segments (Figure 10 ). However, if we assume that all interactions are governed by elasticity, then $\sigma_{l} L=\mu b \approx$ constant and the 'force' $\sigma_{l} L$ opposed by an SFT is independent of the dislocation line length. As Figure 10 shows, this relation is satisfied to a good degree of accuracy for the two segment lengths tried, 70 and $150 \mathrm{~nm}: \sigma_{150 \mathrm{~nm}} / \sigma_{70 \mathrm{~nm}}=0.42 \approx 70 / 150=0.47$. In other words, what these calculations suggest is the existence of a scaling behaviour of the type $\sigma / \sigma^{\prime}=L^{\prime} / L$, indicating that local curvature effects at or near the SFT are negligible compared to the line tension curvature. Obviously, this scaling will break down for dislocation segment lengths of the order of the SFT size.

\subsubsection{Glide and SFT-facet yield curve}

The results presented above have been obtained with stress resolved on the glide plane only. All the processes occurring on SFT $\{111\}$ facets - other than at the base, which sees the same resolved stress as the glide plane - are driven by spontaneous reactions among partial dislocations. It is reasonable to assume, then, that the dislocation-SFT interaction process can be assisted by applying stress on the facet plane. Here we present a simple 


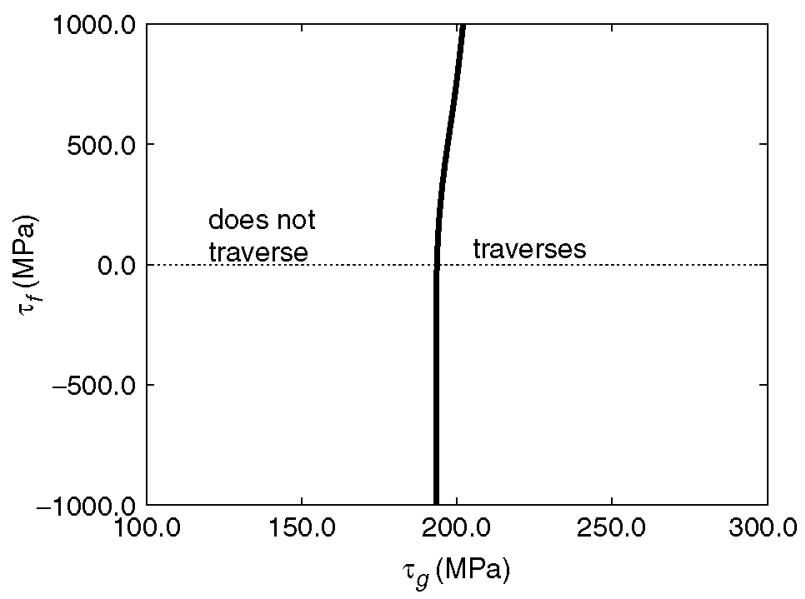

Figure 11. $\tau_{f}-\tau_{g}$ yield curve for a screw dislocation traversing a $4.6-\mathrm{nm}$ SFT. The stress space is divided into two regions, namely the one labelled 'does not traverse', where the dislocation is unable to cut through the SFT, and that labelled 'traverses'. The strength of the stacking fault tetrahedron is practically independent of the stress applied on the SFT facet plane.

analysis to obtain the yield curve of the screw dislocation-tetrahedron interaction at a prescribed height $h / h_{0}=0.47$ for the face-on reaction. We have explored the facet, $\tau_{f}$, and glide, $\tau_{g}$, stress space for mutually orthogonal stress tensors, i.e. the Peach-Köhler force resulting from one stress tensor does not have a component resolved on the other's plane.

This yield curve is shown in Figure 11. Negative values of $\tau_{g}$ indicate a glide force towards the tetrahedron, whereas $\tau_{f}>0$ indicates a downward force resolved on the SFT facet. The stress space is divided into two regions, namely the one labelled 'does not traverse', where the combined stress is not sufficient to force the dislocation through the SFT, and that labelled 'traverses'. In the lower stress quadrant $\left(\tau_{g}>0, \tau_{f}<0\right)$, the strength of the stacking fault tetrahedron to dislocation passage is strictly independent of the stress applied on the SFT facet plane. For $\tau_{f}>0$, the yield curve develops a very slight positive slope, indicating that more glide stress is required to overcome the contrary force resulting from high values of $\tau_{f}$. As we have mentioned earlier, the primary effect of $\tau_{f}$ is to control the transition height from a reaction to an Orowan-loop governed mechanism.

As a good self-consistency check, for $\tau_{f}=0$, we recover the value of $\tau=\tau_{g} \approx 193 \mathrm{MPa}$ presented in Figure 9 for $h / h_{0}=0.47$.

\subsubsection{SFT size effects}

Next we turn to the study of SFT size effects on screw dislocation-SFT interactions. We have carried out simulations for 2.5 and $3.6-\mathrm{nm}$ tetrahedra in the same fashion as in Section 3.2.1 for $h_{0}=4.7 \mathrm{~nm}$. Results are shown in Figure 12, where the strengths for each case as a function of the normalized cutting height are plotted.

Several interesting results emanate from the graphs shown in the figure. First, there is a direct correlation between SFT size and strength. At the base, the 4.7-nm tetrahedron is about $23 \%$ stronger than the $2.5-\mathrm{nm}$ one, and approximately $10 \%$ stronger than the 3.6-nm SFT. The dependence of the basal strength with SFT size is given in the inset 


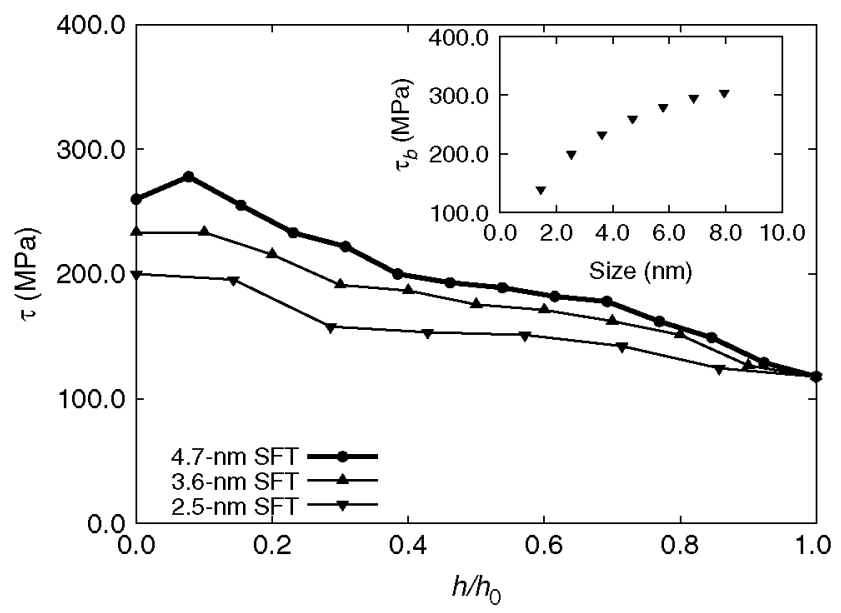

Figure 12. SFT strength as a function of normalized cutting height for three SFT sizes. Inset: basal strength as a function of SFT size.

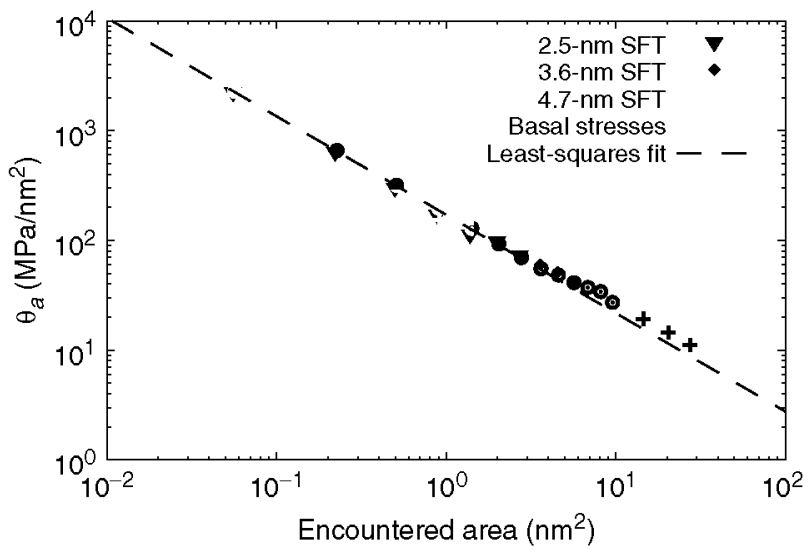

Figure 13. Variation of the stress per unit triangular area, $\theta_{a}$, with the area, $a$, encountered by a screw dislocation inside stacking-fault tetrahedra of several sizes. The curves show that the SFT strength is independent of the tetrahedron size, and depends solely on the area intersected by the dislocation glide plane. The basal stresses are taken from the inset to Figure 12. The least-squares fit to the data is $\theta_{a}(a)=171.02 / a^{0.90}$.

to Figure 12. However, further analysis reveals that the dislocation-SFT interaction stress depends only on the area of the SFT intersected by the dislocation glide plane. This means that the SFT size is not the truly relevant parameter to define the SFT strength: equal triangular areas encountered in different-size tetrahedra give rise to the same response. This dependence gives rise to the self-similar behaviour shown in Figure 13, wherein the stress per unit area, $\theta_{a}$, required to cut the tetrahedron as a function of the 'encountered' area, $a$, is plotted. The results can be conveniently cast into an analytical power law of the type $\theta_{a}(a)=\mathrm{A} a^{n}$, where $\mathrm{A}$ and $n$ are constants, $a=(\sqrt{3} / 4) \ell^{2}$ is the triangular area intersected by the dislocation glide plane, and $\ell=h_{0}-h$ is the size of the 
intersected triangle. This universality is surprising if we keep in mind that the interaction mechanism is not necessarily the same for all the data points shown in the figure. A leastsquares fit to the data from the three SFT sizes explored gives $\theta_{a}(\mathrm{a})=171.02 \mathrm{a}^{-0.90}$, very close to an $a^{-1}$ behaviour. As the figure shows, the agreement between the fit and the calculated data is remarkable in the entire range of areas explored.

Second, all three absolute curves converge to the same apex stress, $\approx 118 \mathrm{MPa}$ if we assume the apex is a resistance point that pins the dislocation, or $86.7 \mathrm{MPa}$ if we only take into account the stress field due to the SFT below. The observed convergence as a function of size suggests that, at the apex, local effects dominate over long-range effects. For the figures we have taken the conservative (upper-bound) value of $118 \mathrm{MPa}$, although, as mentioned above, it is reasonable to expect that the actual strength lies between this value and the lower estimate of $86.7 \mathrm{MPa}$.

All this information may now be used to derive a simple rule to calculate the strength of a tetrahedron of arbitrary size to screw dislocation passage face-on. The only parameters needed to define these interactions are now the size-independent apex strength and the global fitting function, also size-independent, given above for the data shown in Figure 12. Evidently, at the apex the fitting function is not defined $(a=0)$, but the apex stress is finite and known, and the $\theta_{a}(a)$ function can be applied all the way up to a prescribed distance from it (e.g. a fraction of a nm):

$$
\begin{gathered}
\theta_{a}=\frac{\tau}{a}=\frac{171.02}{a^{0.90}} \theta \\
\tau=171.02 a^{0.10}
\end{gathered}
$$

where stresses are given in $\mathrm{MPa}$ and areas in $\mathrm{nm}^{2}$. Equation (5), together with $a=(\sqrt{3} / 4)\left(h_{0}-h\right)^{2}$ suffices to provide a closed model for the strength of SFTs in the $2.5-4.7-\mathrm{nm}$ size range for the case of $70-\mathrm{nm}$ screw dislocation.

\section{Discussion}

\subsection{Methodology}

First, let us discuss some of the strengths and weaknesses of our DD methodology with respect to other techniques. From a qualitative point of view, our DD simulations are representative of low strain-rate experiments $\left(\sim 10^{-5} \mathrm{~s}^{-1}\right)$, where dislocations move sufficiently slow to allow dislocation reactions to occur quasi-statically. In fact, although analytically cumbersome for non-trivial geometries, in principle these interactions could be extracted directly from static elasticity theory, as it has been done Conversely, most atomistic simulations operate at strain rates of the order of $10^{6} \mathrm{~s}^{-1}$ and higher, potentially resulting in high dislocation velocities. In such cases, dynamic effects dominate over stress field coupling, and trivial interactions, such as simple shear of SFTs, are typically observed

On the other hand, our methodology lacks finite displacements, which are important if one wants to model elementary atomistic mechanisms. For example, our simulations cannot explicitly account for the $b$-magnitude shear carried by dislocations as they glide and interact with obstacles. Although the impact of neglecting this shear on the nature and time-scale of the dislocation reactions observed is unclear, it may become important for 
the long-term evolution of the resulting SFT structures. Another important issue is the possibility for vacancy removal in our simulations, leading to truly smaller SFT sizes. Evidently, atomic-wise processes such as vacancy diffusion and reorganization cannot be taken into account in DD. However, there is a parameter in our methodology which can be critically tuned to model processes of vacancy absorption by dislocation segments: the climb drag coefficient, $B_{c}$, in Equation (3). Calibrating this parameter in the proximity of stacking-fault tetrahedra may be useful to enrich our interaction physics and capture mechanisms such as those observed by Osetsky

The structures shown in Figures 5 and 8 are metastable, and could either (i) give rise to reconstructed, perfect SFTs, or (ii) react into some stable, but not perfect, structures, or (iii) remain in their metastable state. It is the power dissipation criterion, used in our methodology to calculate the relative likelihood of a given transition that determines which case is favoured. Of course, the detailed energetics of these transitions are not included in DD, and a full examination of the stability of these structures can only truly be made using atomistic methods. However, we emphasize that our study is one of SFT strength, not SFT stability under dislocation interactions (for which MD is no doubt much better suited). Because of the conservation of the vacancy size of the SFT in DD, however, option (i) is generally a preferred reaction pathway in the absence of applied stress.

Recently, several atomistic simulations of interactions between screw dislocations and stacking-fault tetrahedra have been carried out quasistatically

Some of these simulations are in excellent agreement with the mechanism showcased in Figure 5, although this mechanism is by no means unique, as we have seen in step (4) of Section 3.2.1. In any case, a common feature shared by all these atomistic studies and our simulations is the impossibility of completely removing the tetrahedron in a single passage. These conclusions stand in contrast with some experimental studies, which generally support the idea of complete SFT removal according to Kimura's model or some variation thereof $[19,20]$. Nevertheless, recent in situ TEM experiments have revealed new mechanisms that are not inconsistent with our simulations

Also, new MD studies provide further evidence that truncated or SFT-like structures left after a first dislocation passage are relatively easier to remove than perfect tetrahedra

The stresses computed here, between 100 and $300 \mathrm{MPa}$, are generally higher than those typically measured experimentally which are of the order of $\lesssim 100 \mathrm{MPa}$ for defect densities consistent with our segment length of $70 \mathrm{~nm}$. However, these critical stresses are obtained from a variety of defects (not all SFTs) in different conditions, most notably finite temperature and dislocation segment length. Indeed, Osetsky et al. have shown that the critical stress displays a marked temperature dependence. Our data are in much better agreement with the MD results, which give values in the range of $\sim 250 \mathrm{MPa}$

This despite the boundary conditions employed, periodic in atomistic studies and fixed end-points in our simulations. The qualitative and quantitative similarities between both approaches thus suggest that MD simulations are acceptable in terms of length-scale, as they would capture the line tension effects that govern the DD simulations.

An obvious advantage of DD is the computational savings with respect to atomistic methods. In general, these savings are the result of a number of assumptions, which reduce the amount of physical information incorporated into the method. With the methodology presented here, we believe that we have achieved a satisfactory compromise among numerical accuracy, computational cost, and generality. Furthermore, one of our most 
important conclusions is the fact that our DD simulations are obtained with a closed model that uses unit input extracted from the atomistic scale. This atomistic information is entirely fundamental in the sense that is not specific to the dislocation-SFT problem, although, as such, it is of course subject to the limitations inherent to atomistic calculations laid out in Section 3.1. Thus, our simulations can be considered 'stand-alone' in the sense that they can be independently compared or added to existing simulation databases involving dislocation-SFT interactions.

\subsection{Implications for irradiated material experiments: shear band formation}

The interaction among gliding dislocations and SFTs (as well as other small irradiationproduced obstacles) is believed to be one of the mechanisms responsible for the formation of defect-free shear bands ('channels') in deformed irradiated metals. However, recent experiments have shown that there still exists a large number of uncertainties regarding the qualitative and quantitative predictions made by models based on dislocation-defect interactions Ghoniem et al. have proposed that local heating may be responsible for the dissolution of SFTs, by emission of individual vacancies that are then absorbed by the dislocation [29]. According to these authors, this results in local dislocation climb and jog formation, which, accumulated over the glide distances available to moving dislocations (e.g. grain size, specimen size, etc.), is what gives rise to the formation of channels. This picture assumes that, similar to the Kimura mechanism

SFTs are completely removed by a single dislocation passage (after some critical angle has been reached). Recent estimates do indeed suggest that an average amount of 1.5 dislocations are needed to remove SFTs from the channels in order to account for the measured shear deformation accumulated in the channels. However, there is very little evidence for this mechanism, both from MD and, now, from DD simulations. Nor is there evidence for the cross-slip mechanism suggested by Hiratani et al. that requires the stressassisted conversion of SFTs into Frank loops This is also the mechanism proposed by Khraishi et al. for the absorption of SFTs, i.e. that they become glissile via a collapse onto a Frank loop before they can be eliminated by the dislocation

Our simulations, on the other hand, may suggest a different scenario, not necessarily opposed to the one proposed by Ghoniem and co-workers, but complementary. Our results indicate that stacking fault tetrahedra are significantly weakened after one dislocation passage, which, although not sufficient to fully destroy the SFT, may shrink it below the standard TEM resolution threshold (it is worth remarking that irradiation-produced SFTs, at $1.5-3.0 \mathrm{~nm}$, are already close to such a threshold to start with). We argue that our mechanisms, characterized solely by partial dislocation reactions, may possibly produce softening locally, triggering strain localization. As a matter of fact, preliminary calculations show that the structures shown in Figures 5(6) and 8(6) represent weaker obstacles to subsequent dislocation passage than the corresponding initial tetrahedra.

The width of the channels, experimentally observed to range between 100 and $200 \mathrm{~nm}$, has been rationalized in terms of (i) recurrent atomic-wise climb over long glide distances and (ii) double cross-slip This presupposes the activation of a single slip plane, which, by successive climb and double cross-slip events, multiplies into other sources on co-planar systems, until, according to Khraishi the back-stress created by dipoles opposing further cross-slip reaches a critical value. However, although we do not 
question the plausibility of these mechanisms, nothing precludes the pre-existence of coplanar dislocation sources across some distance (the 'channel' width) acting in unison. This was in fact observed by Robertson and co-workers dislocations nucleate heterogeneously

in polycrystalline $\mathrm{Cu}$, where at crack tips or grain boundaries on multiple planes. In addition, no double cross-slip is observed in our simulations. In fact, the activation energies for cross-slip have been calculated atomistically to be rather high, of the order of $2.5 \mathrm{eV}$. In another publication we have provided estimates of the critical cross-slip stress in a variety of loading conditions, and found it to be a non-negligible fraction of the shear modulus. This may limit the prolificness for cross-slip required in these models, even in the presence of high local stress concentrations in the vicinity of radiation-induced defects. The question, in our minds, is why only a range of co-planar sources $(100-200 \mathrm{~nm}$, again the 'channel' width) is activated. We do not provide information here to answer this, and more research is needed in order to shed more light on these and other aspects of irradiated materials plasticity.

\subsection{Large-scale simulations by model homogenization}

The final point that we want to touch upon is the coarsening of these results to be used in large-scale DD plasticity simulations. The short-range nature of many of the partial dislocation reactions observed in our simulations drives the integration time-step below one picosecond in some cases, which precludes the direct use of our technique for largescale simulations in the, e.g. $\varepsilon \geq 5-10 \%$ regime. In this regard, the modifications pointed out by Martinez et al. to self-consistently reduce multi-partial dislocation reactions to equivalent perfect dislocation reactions may represent a step towards coarsening the model to expedite the calculations

The overarching question is how to condense our most important findings, namely, (i), that the strength of a stacking-fault tetrahedron to screw dislocations is independent of its size, and, (ii), that this strength is governed purely by line tension effects, into higher-level hierarchical models. Per (i), our results indicate that the parameter that governs SFT strength is the triangular cross section intersected by the glide plane, and that equal areas intersected in different size tetrahedra give rise to the same mechanical response. Point (ii) suggests that the interaction mechanisms are simply internal transformations necessary to accommodate the line tension built up into the outer dislocation segments when they are stress-loaded. As we have mentioned, this behaviour is expected to break down for very short line lengths (of the order of the tetrahedron size).

To apply additional levels of homogenization to these results is no easy task, as real plasticity in irradiated materials is a concoction of different processes involving multiple SFT orientations and sizes, dislocation line lengths and characters, etc. As we have seen in Section 3.3.2, the product $\tau L$ is conserved for any value of $L$ :

$$
\tau L=171.02 a^{0.10}[\mathrm{MPa}] \cdot 70[\mathrm{~nm}]=11971.4 a^{0.10}
$$

This expression relates the strength of a given stacking-fault tetrahedron with the size of a Frank-Read source and the areal section of the SFT intersected by the glide plane on which the source operates. This simple model is an example of how our results can be elevated into coarser techniques that do not include the fine resolution employed in our calculations. Nevertheless, much work remains before this information can be 
integrated into continuum plasticity models based on effective dislocation densities that can supersede the more phenomenological models used in previous works Ultimately, for meaningful simulations, other mechanisms, such as interactions with interstitial Frank loops or dislocation decoration $[65,66]$, ought to be included.

As discussed in Section 4.1, our methodology now contains the ingredients required to accomplish this, namely, computational expeditiousness balanced with atomic-like resolution.

\section{Conclusions}

- We have carried out a comprehensive dislocation dynamics study of SFT stability and dislocation-SFT interactions in fcc $\mathrm{Cu}$.

- Where the comparisons are meaningful, our DD simulations have been benchmarked against and validated with atomistic and experimental data, with reasonable agreement. However, our DD simulations can be considered independent of prior atomistic and DD studies, as the methodology is not influenced by conclusions previously extracted from computational or experimental works, and stand by themselves as an independent source of data.

- We have computed the stress required by a dislocation in fcc $\mathrm{Cu}$ to traverse a stacking fault tetrahedron as a function of SFT size and distance between the SFT base and the glide plane.

- We have identified the interaction mechanisms in each case and analyzed the resulting SFT structures. We see that screw dislocations require higher stresses to traverse the defect when they are close to the base, and that there exist two interaction regimes, namely, reactions vs. Orowan loops, as a function of the interaction height. In the former, dislocations are effective in damaging the SFT, although we do not see complete absorption in any of the cases studied.

- We have obtained a power law that gives the SFT strength as a function of the area intersected between the SFT and the dislocation glide plane. This law is strictly valid for $70-\mathrm{nm}$ screw dislocation segments interacting with $2.5-4.7-\mathrm{nm}$ SFTs face-on.

- We observe that the results are very sensitive to the initial dislocation line length, which suggests that a line tension model could suffice to capture these processes, and that results could differ substantially depending on the internal microstructure.

- Two new kinds of Burgers vectors have been observed, namely, $(1 / 6)\langle 013\rangle$, which is topologically similar to a stair-rod, and $(1 / 6)\langle 321\rangle$, akin to a Hirth dislocation. Although these dislocations are unstable, they can occur temporarily, stabilized by shear stress. 


\section{Notes}

1. Due to the curvature, the line sense is actually reversed, resulting in a BA segment, rather than the original $\mathrm{AB}$.

2. Shearing is characterized by $f_{i}>f^{*}, i=1, \ldots, N_{s}$ where $N_{s}$ is the number of nodes in contact with a stacking fault surface.

\section{References}

K.C. Russell, Prog. Mater. Sci. 28 (1984) p.229.

B.N. Singh, A. Horsewell, P. Toft et al., J. Nucl. Mater. 224 (1995) p.131.

M. Victoria, N. Baluc, C. Bailat et al., J. Nucl. Mater. 276 (2000) p.114.

B.N. Singh and S.J. Zinkle, J. Nucl. Mater. 206 (1993) p.212.

Y. Dai, X. Jia, J.C. Chen et al., J. Nucl. Mater. 296 (2001) p.174.

S.J. Zinkle and R.L. Sindelar, J. Nucl. Mater. 155-157B (1988) p.1196.

R. Schäublin, Z. Yao, N. Baluc et al., Phil. Mag. 85 (2005a) p.769.

S.J. Zinkle and L.L. Snead, J. Nucl. Mater. 225 (1995) p.123.

M. Eldrup and B.N. Singh, J. Nucl. Mater. 276 (2000) p.269.

Y. Satoh, I. Ishida, T. Yoshiie et al., J. Nucl. Mater. 155-157 (1988) p.443.

T.S. Byun and K. Farrell, Acta Mat. 52 (2004) p.1597.

N. Hashimoto, B.T.S.,K. Farrell, S.J. Zinkle et al., J. Nucl. Mater. 329-333 (2004) p.947.

R.P. Tucker, M.S. Wechsler and S.M. Ohr, J. Appl. Phys. 40 (1969) p.400.

M.S. Wechsler, Tech. Rep. CONF-711047-1, Ames Lab., Iowa (1971).

P. de Almeida and M. Victoria, Solid State Comm. 125 (2003) p.195.

F. Onimus, I. Monnet, J. Béchade et al., J. Nucl. Mater. 328 (2004) p.165.

Z. Yao, R. Schäublin and V.M. Victoria, J. Nucl. Mater. 307-311 (2002) p.374.

S.J. Zinkle and B.N. Singh, J. Nucl. Mater. 283-287 (2000) p.306.

Y. Matsukawa and S.J. Zinkle, J. Nucl. Mater. 329-333 (2004) p.919.

R. Schäublin, Z. Yao, P. Spätig et al., Mater. Sci. \& Eng. A 400-401 (2005b) p.251.

J.S. Robach, I.M. Robertson, H.J. Lee et al., Acta Mater. 54 (2006) p.1679.

Y. Matsukawa, Y.N. Osetsky, R.E. Stoller et al., J. Nucl. Mater. 351 (2006) p.285.

B.D. Wirth, V.V. Bulatov and T. Díaz de la Rubia, J. Eng. Mater. \& Technol.-Trans. ASME 124 (2002) p.329.

D. Rodney, Nucl. Inst. \& Meth. Phys. Res. B 228 (2005) p.100.

Y.N. Osetsky, R.E. Stoller, D. Rodney et al., Mater. Sci. \& Eng. A 400-401 (2005) p.370.

P. Szelestey, M. Patriarca and K. Kaski, Modelling Simul. Mater. Sci. Eng. 13 (2005) p.541.

Y.N. Osetsky, Y. Matsukawa, R.E. Stoller et al., Phil. Mag. Lett. 86 (2006a) p.511.

H.J. Lee, J.H. Shim and B.D. Wirth, J. Mater. Res. 22 (2007) p.2758.

N.M. Ghoniem, S.-H. Tong, B.N. Singh et al., Phil. Mag. A 81 (2001) p.2743.

N.M. Ghoniem, S.H. Tong, J. Huang et al., J. Nucl. Mater. 307-311 (2002) p.843.

T.A. Khraishi, H.M. Zbib, T. Diaz de la Rubia et al., Metall. Mater. Trans. B 33B (2002) p.285.

L.Z. Sun, N.M. Ghoniem and Z.Q. Wang, Mater. Sci. Eng. A 309-310 (2001) p.178.

M. Hiratani, H.M. Zbib and B.D. Wirth, Phil. Mag. A 82 (2002) p.2709.

M. Hiratani, V.V. Bulatov and H.M. Zbib, J. Nucl. Mater. 329-333 (2004) p.1103.

E. Martínez, J. Marian, A. Arsenlis et al., J. Mech. Phys. Solids (2007) p.869.

A. Arsenlis, W. Cai, M. Tang et al., Modell. Simul. Mater. Sci. Eng 15 (2007) p.553. 
P. Müllner and P.J. Ferreira, Phil. Mag. Lett. 73 (1996) p.289.

J.P. Hirth and J. Lothe, Theory of Dislocations, Krieger Publishing Company, Malabar, Florida, 1992.

Z.-H. Jin, P. Gumbsch, E. Ma et al., Scripta Mater. 54 (2006) p.1163.

H. Kimura, D. Kuhlmann-Wilsdorf and R. Maddin, Appl. Phys. Lett. 3 (1963) p.4.

W. Westdorp, R. Maddin and H. Kimura, Acta Metall. 12 (1964) p.495.

M. Kiritani, Mater. Chem. Phys. 50 (1997) p.133.

Q.F. Guan, L. Pan, H. Zou et al., J. Mater. Sci. 39 (2004) p.6349.

E.J. Savino and R.C. Perrin, J. Phys. F: Metal Phys. 4 (1974) p.1889.

K. Nordlund and F. Gao, Appl. Phys. Lett. 74 (1999) p.2720.

B.D. Wirth, V.V. Bulatov and T. Díazdela Rubia, J. Nucl. Mater. 283 (2000) p.773.

R.E. Voskoboinikov, Y.N. Osetsky and D.J. Bacon, Nucl. Inst. Meth. Phys. Res. B 242 (2006) p.68.

B.P. Uberuaga, A.F. Voter and R. Hoagland, private communication (2007).

J. Silcox and P.B. Hirsch, Phil. Mag. 4 (1959) p.72.

H. Kimura and R. Maddin, in Proceedings of an International Conference, R. Cotterill, ed., Argonne National Laboratory Academic Press, New York, 1965, p.319.

E. Johnson and P.E. Hirsch, Phil. Mag. A 43 (1981) p.157.

J.S. Robach, I.M. Robertson, B.D. Wirth et al., Phil. Mag. 83 (2003) p.955.

Y.N. Osetsky, D. Rodney and D.J. Bacon, Phil. Mag. 86 (2006) p.2295.

T. Nogaret, C. Robertson and D. Rodney, Phil. Mag. 87 (2007) p.945.

L. Saintoyant, H.J. Lee and B.D. Wirth, J. Nucl. Mater. 361 (2007) p.206.

D.J. Edwards, B.N. Singh and J.B. Bilde-Sorensen, J. Nucl. Mater. 342 (2005) p.164.

N. Hashimoto, T.S. Byun and K. Farrell, J. Nucl. Mater. 351 (2006) p.295.

B.N. Singh, A.J.E. Foreman and H. Trinkaus, J. Nucl. Mater. 249 (1997) p.103.

M. Victoria, private communication.

I.M Robertson, A. Beaudoin, K. Al-Fadhalah et al., Mater. Sci. Eng. A 400-401 (2005) p.245.

D.J. Edwards and B.N. Singh, J. Nucl. Mater. 329-333 (2004) p.1072.

S. Rao, T.A. Parthasarathy and C. Woodward, Phil. Mag. A 79 (1167) p.1999.

D. Mordehai, I. Kelson and G. Makov, Mater. Sci. Eng. A 400-401 (2005) p.37.

A. Arsenlis, B.D. Wirth and M. Rhee, Phil. Mag. 84 (2004) p.3617.

L.Z. Sun, N.M. Ghoniem, S.-H. Tong et al., J. Nucl. Mater. 283-287 (2000) p.741.

H. Trinkaus, B.N. Singh and A.J.E. Foreman, J. Nucl. Mater. 249 (1997) p.91.

M.R. Surowiec, Phys. Stat. Sol. (a) 122 (1990) p.K15.

\section{Appendix A: Face-on basal mechanisms}

\section{A.1. AB dislocation}

Figure A1 shows four different frames of the face-on interaction mechanism. There is a direct correspondence between the frames in the figure and the itemized mechanism below. The process starts with an $\mathrm{AB}$ dislocation, dissociated into a pair of Shockley partials $\mathrm{A} \delta$ (leading) and $\delta \mathrm{B}$, gliding on the $(d)$ plane at some short distance from the SFT.

(1) The leading partial reacts with the basal stair-rod $\delta \gamma$, and creates a Shockley A $\gamma$ that is glissile on the tetrahedron's facet $(c)$ :

$$
\mathrm{A} \delta+\delta \gamma \rightarrow \mathrm{A} \gamma
$$

For its part, this partial curves on (c) and comes into contact with the $\gamma \beta$ stair rod:

$$
\mathrm{A} \gamma+\gamma \beta \rightarrow \mathrm{A} \beta
$$



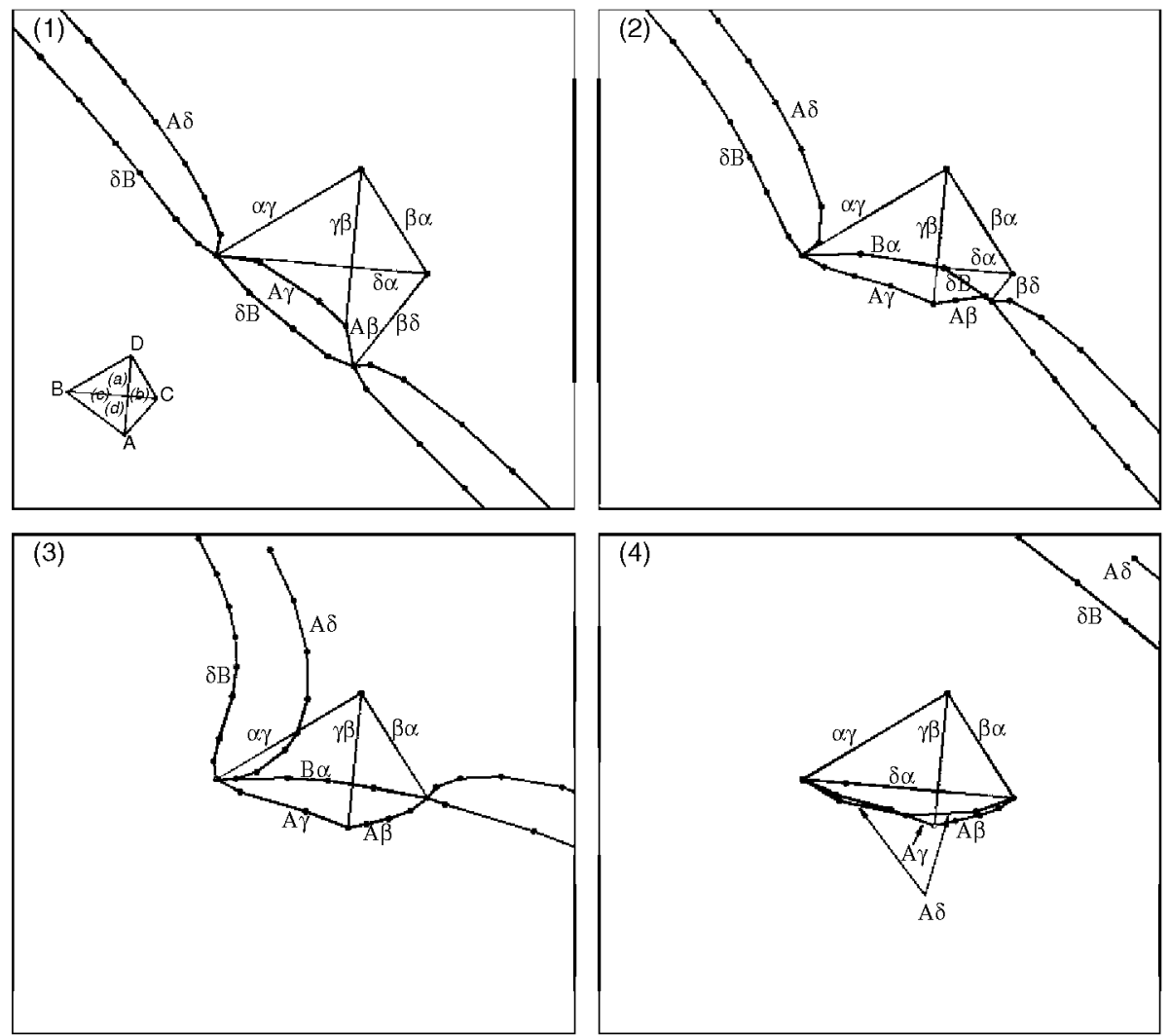

Figure A1. Base mechanism for an $\mathrm{AB}$ screw dislocation interacting with a 4.7-nm SFT face-on. Segments are colour-coded according to their Burgers vector: in blue Shockley partials and in green stair-rod dislocations. The inset in frame (1) shows the orientation of the Thompson tetrahedron used for this set of simulations. For colour version, see online.

(2) After the $\delta \gamma$ stair-rod is removed, the trailing partial sweeps the base, removing the stacking fault after itself. When it reaches the back face $(a)$, it reacts with $\delta \alpha$, giving rise to a $\mathrm{B} \alpha$ Shockley:

$$
\delta \mathrm{B}+\delta \alpha \rightarrow \mathrm{B} \alpha
$$

At the same time, $\mathrm{A} \beta$ glides on $(b)$, removing more stacking fault.

(3) With the removal of all the basal stair-rods the SFT appears as truncated, with its original base completely gone.

(4) After the leading and trailing partials curve around the defect, $\delta \mathrm{B}$ collapses onto $\mathrm{B} \alpha$, reconstructing the original $\delta \alpha$ dislocation:

$$
\delta \mathrm{B}+\mathrm{B} \alpha \rightarrow \delta \alpha
$$

\section{A.2. BA dislocation}

The mechanism is shown in Figure A2. In this case we have a BA dislocation dissociated into $\delta \mathrm{A}$ (leading) and $\mathrm{B} \delta$. The simulation starts with the dislocation placed on the $(d)$ plane at a short 

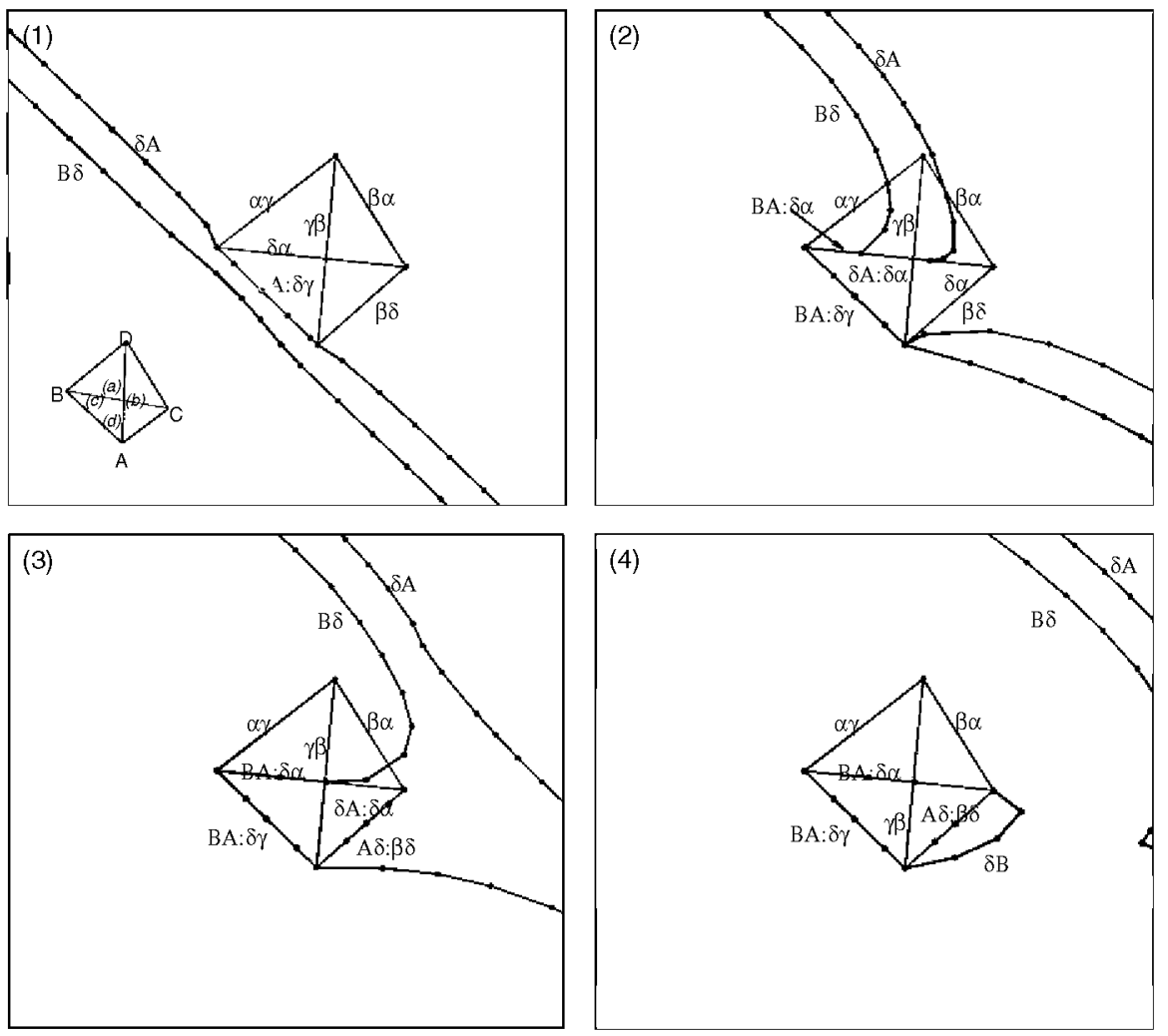

Figure A2. Base mechanism for a BA screw dislocation interacting with a 4.7-nm SFT face-on. Segments are colour-coded according to their Burgers vector: in blue Shockley partials, in green stair-rods, and in orange Hirth dislocations and other non-crystallographic Burgers vectors. The inset in frame (1) shows the orientation of the Thompson tetrahedron used for this set of simulations. For colour version, see online.

distance from the SFT base. The subsequent reaction mechanism is as follows:

(1) The leading partial reacts with the basal stair-rod $\delta \gamma$, giving rise to a sessile $\delta \mathrm{A}: \delta \gamma$ across the entire base:

$$
\delta \mathrm{A}+\delta \gamma \rightarrow \delta \mathrm{A}: \delta \gamma
$$

The product dislocation corresponds to a Burgers vector of $(1 / 6)[0 \overline{3} 1]$, which is unstable from an elastic energy point of view and would thus be expected to dissociate when stress ceases to be applied. Interestingly, this type of dislocation has been proposed in the literature to hypothesize a new type of dislocation lock in fec crystals

(2) The segment of the dislocation to the left of the SFT reacts with the $\delta \alpha$ stair rod, first giving rise to a Hirth-type segment via reaction with the leading partial:

$$
\delta \mathrm{A}+\delta \alpha \rightarrow \delta \mathrm{A}: \delta \alpha
$$

i.e. $\delta \mathrm{A}: \delta \alpha \equiv(1 / 3)[010]$, which is energetically favourable. The trailing partial then collapses onto this Hirth segment, producing a BA : $\delta \alpha$ :

$$
\mathrm{B} \delta+\delta \mathrm{A}: \delta \alpha \rightarrow \mathrm{BA}: \delta \alpha
$$


This BA: $\delta \alpha$ dislocation corresponds to a Burgers vector of $(1 / 6)$ which renders this reaction endoergic as well.

(3) A similar process is seen for the other arm of the screw dislocation, where the following reaction takes place (note that, due to the line curvature, the initial BA dislocation reverses its sign):

$$
\mathrm{A} \delta+\beta \delta \rightarrow \mathrm{A} \delta: \beta \delta
$$

which has a Burgers vector of $(1 / 6) \quad$ After sweeping the entire edge, the leading partial detaches.

(4) The trailing partial detaches converting the entire left edge of the base to a BA: $\delta \alpha$ dislocation and leaving an independent $\delta \mathrm{B}$ segment on the right-hand side, which, interestingly, does not collapse onto the existing $\mathrm{A} \delta: \beta \delta$.

This mechanism is the basal equivalent to the Orowan loop process observed in Section 3.2.1. As we have seen, all the segments comprising the triangular perimeter of the loop are energetically unfavourable and expected to dissolve in the absence of applied stress. These stress-stabilized dislocations are topologically plausible, and physically possible within the framework of linear elasticity. However, little is known about them - explicit mentions to them are virtually absent in the literature - and here we simply note their existence. (1/6) $\langle 013\rangle$ dislocations are the high-energy equivalents of traditional $(1 / 6)\langle 110\rangle$ stair-rod dislocation, i.e. they provide the lattice disregistry required to rotate a stacking fault from one $\{111\}$ plane to another. For their part, $(1 / 6)\langle 123\rangle$ dislocations resemble $(1 / 3)\langle 001\rangle$ Hirth dislocations in that they can act as the physical limit of three stacking faults.

\section{Appendix B: Edge-on basal mechanisms}

\section{B.1. AB dislocation}

The basal mechanism when the dislocation impinges edge-on is shown in Figure $\mathrm{B} 1$. The interaction process proceeds as follows:

(1) The leading A $\delta$ partial is locally pinned at the SFT vertex, making it curve around until it comes in contact with the edges $\delta \alpha$ and $\beta \delta$.

(2) The reaction of the leading partial (which becomes $\delta \mathrm{A}$ due to curvature) with the $\beta \delta$ edge results into a $\beta$ A partial:

$$
\delta \mathrm{A}+\beta \delta \rightarrow \beta \mathrm{A}
$$

The trailing partial penetrates the SFT base, sweeping the stacking fault in its wake, until it reaches the $\delta \alpha$ edge, giving rise to a $\mathrm{B} \alpha$ partial. This partial reacts in turn with the curved portion of the leading partial, locally resulting in a Hirth-type dislocation:

$$
\begin{aligned}
& \delta \mathrm{B}+\delta \alpha \rightarrow \mathrm{B} \alpha \\
& \mathrm{A} \delta+\mathrm{B} \alpha \rightarrow \mathrm{AB}: \delta \alpha
\end{aligned}
$$

(3) The newly-created $\mathrm{B} \alpha$ can glide (up) on the (a) plane, which it does, removing the stacking fault until it stops repelled by the $\beta \alpha$ stair $-\operatorname{rod}$. When $\mathrm{B} \alpha$ glides up on $(a)$, the leading partial $\mathrm{A} \delta$ is able to glide unimpeded on the basal plane until it encounters $\beta \mathrm{A}$, giving rise to a reconstructed $\beta \delta$ :

$$
\mathrm{A} \delta+\beta \mathrm{A} \rightarrow \beta \delta
$$

For its part, the section of the trailing partial that had penetrated the base reaches the back edge $\delta \gamma$ giving rise (after correcting the sign for curvature) to a $\mathrm{B} \gamma$ partial:

$$
\mathrm{B} \delta+\delta \gamma \rightarrow \mathrm{B} \gamma
$$

Frame (3) in Figure B1 marks the maximum degree of SFT damage of the entire process. 

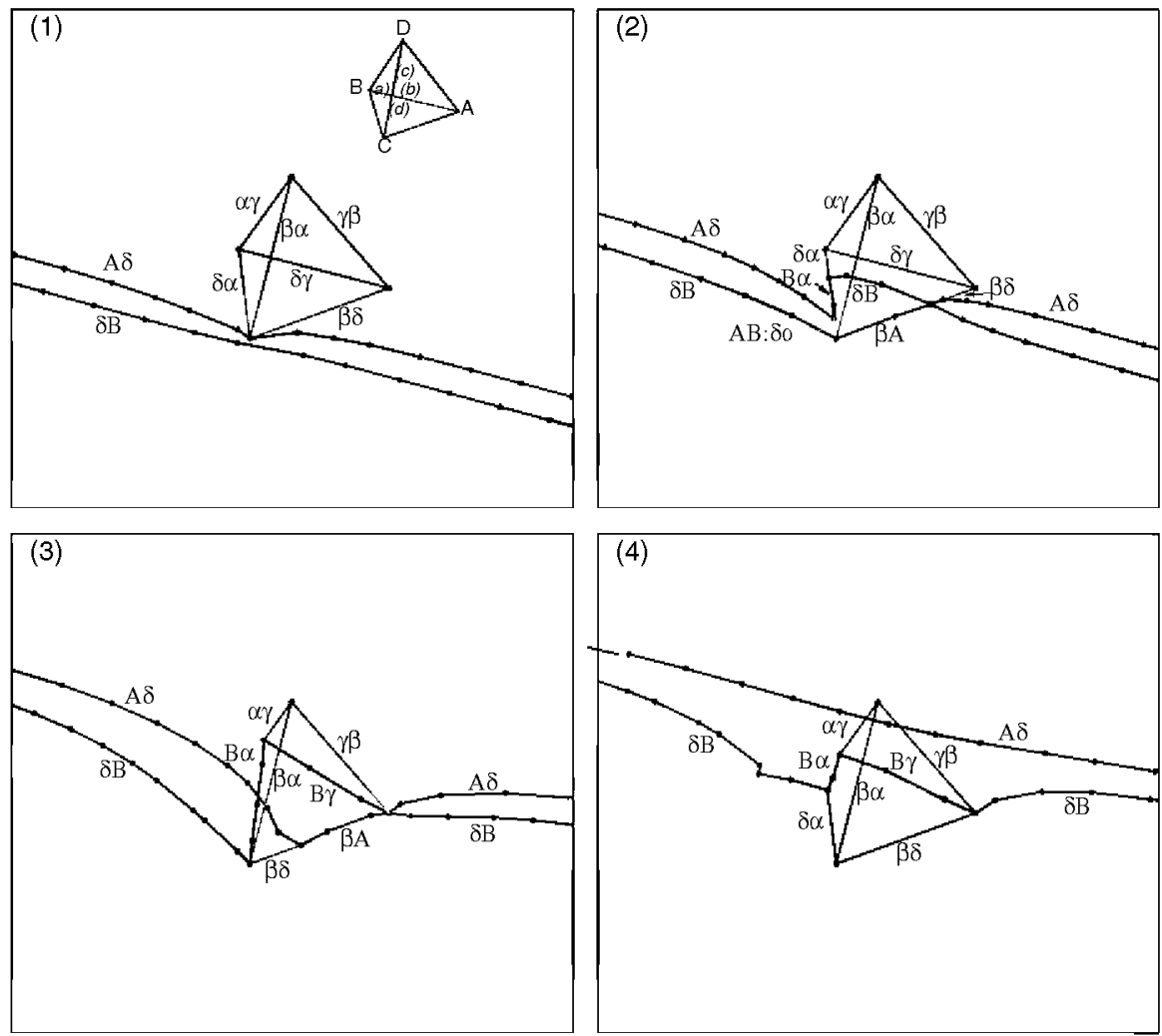

Figure B1. Base mechanism for a screw dislocation interacting with a 4.7-nm SFT edge-on. Segments are colour-coded according to their Burgers vector: in blue Shockley partials, in green stair-rod dislocations, and in orange, Hirth-type and other dislocations. The inset in frame (1) shows the orientation of the Thompson tetrahedron used for this set of simulations. For colour version, see online.

(4) After $\mathrm{A} \delta$ and $\beta \mathrm{A}$ zip and reconstruct the $\beta \delta$ edge entirely, the leading partial detaches. Meanwhile, the trailing partial reacts with the $\mathrm{B} \delta$ Shockley, reconstructing the $\delta \alpha$ edge. Finally, when $\delta \mathrm{B}$ curves around the back face of the SFT it reacts with $\mathrm{B} \gamma$, leaving the original tetrahedron intact.

\section{B.2. BA dislocation}

When the Burgers vector's direction is reversed the interaction mechanism, shown in Figure A2 changes substantially:

(1) On the left side of the SFT, the leading partial reacts with the $\delta \alpha$ stair-rod, giving rise to the following Hirth segment:

$$
\delta \mathrm{A}+\delta \alpha \rightarrow \delta \mathrm{A}: \delta \alpha
$$

which has a $(1 / 3) \quad$ Burgers vector. When the trailing partial collapses onto this segment, it forms a $(1 / 6)$ dislocation:

$$
\mathrm{B} \delta+\delta \mathrm{A}: \delta \alpha \rightarrow \mathrm{BA}: \delta \alpha .
$$



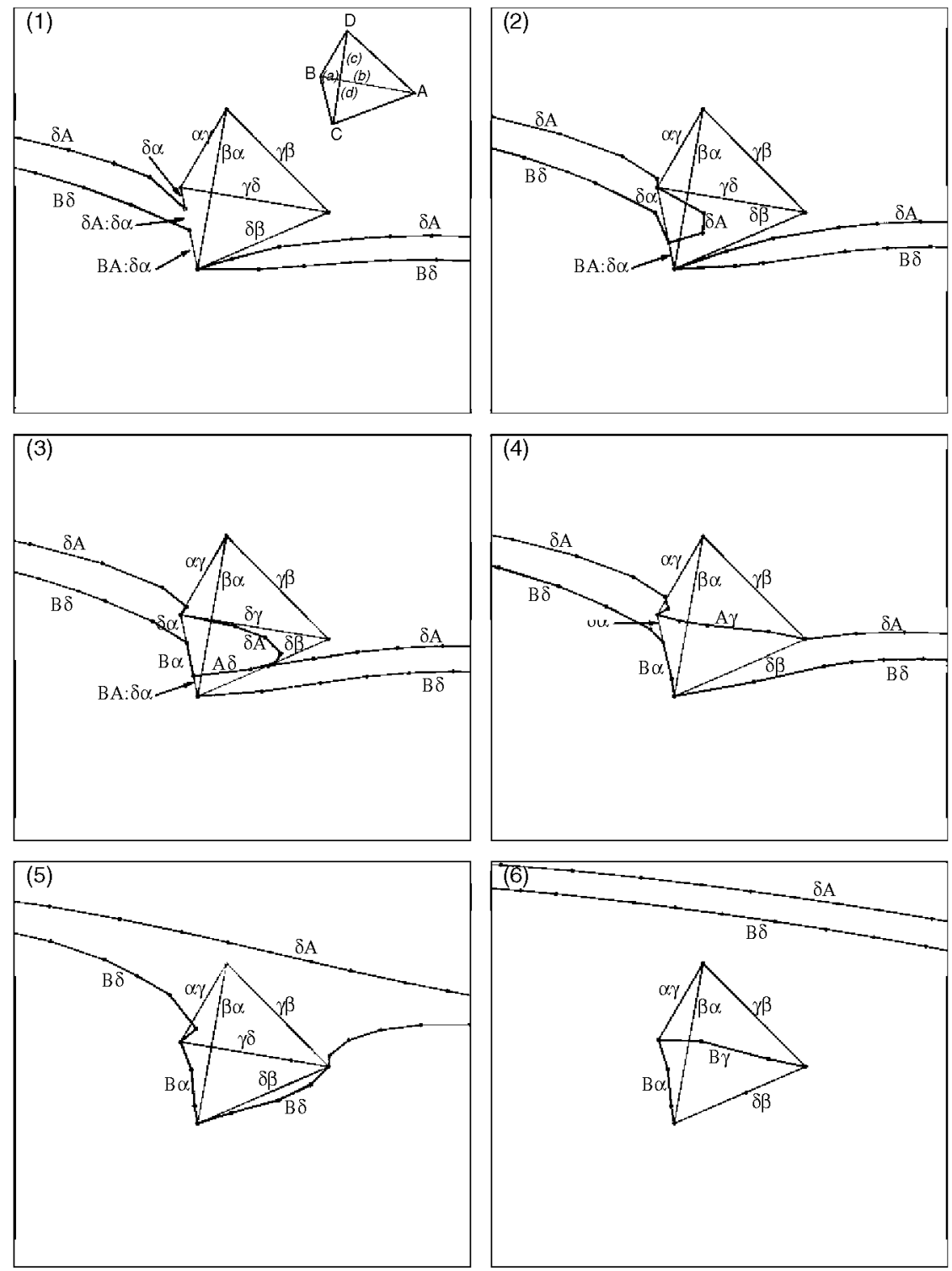

Figure B2. Base mechanism for a screw dislocation interacting with a 4.7-nm SFT edge-on. Segments are colour-coded according to their Burgers vector: in blue Shockley partials, in green stair-rod dislocations, and in orange, Hirth and other dislocations. The inset in frame (1) shows the orientation of the Thompson tetrahedron used for this set of simulations. For colour version, see online.

(2) The Hirth dislocation now suffers an interesting transformation: it re-dissociates into $\delta \mathrm{A}$ and $\delta \alpha$ inside the base of the tetrahedron. This results in the Shockley partial removing the stacking fault as it glides inward, attracted by the $\beta \delta$ stair-rod of the opposite side. In terms of Equation (2) this dissociation involves a reversal of the $\gamma \mathbf{n}$ vector associated with the original $\delta \mathrm{A}$ and $\delta \alpha$ dislocations. 
(3) The inner $\delta \mathrm{A}$ partial curves about (changing its sign), reacting with $\mathrm{BA}: \delta \alpha$ to give a Shockley partial that is glissile on $(a)$ :

$$
\mathrm{A} \delta+\mathrm{BA}: \delta \alpha \rightarrow \mathrm{B} \alpha
$$

On the right side of the SFT, the leading partial bears down on $\beta \delta$ leading to the following reaction:

$$
\delta \mathrm{A}+\delta \beta \rightarrow \delta \mathrm{A}: \delta \beta
$$

which is a high-energy $(1 / 6)[\overline{1} 30]$ dislocation.

(4) The inner $\delta \mathrm{A}$ partial now reacts with the three base edges as follows. On the left side the first reaction of the previous step is completed. On the right-hand side it reacts (as $A \delta$ ) with the product of the second reaction of the previous step:

$$
\mathrm{A} \delta+\delta \mathrm{A}: \delta \beta \rightarrow \delta \beta
$$

On the back edge, the following reaction takes place:

$$
\delta \mathrm{A}+\gamma \delta \rightarrow \gamma \mathrm{A}
$$

which is a Shockley partial capable of gliding on the back face $(c)$. With these set of reactions, the $(d)$ stacking fault (the original base) is completely removed.

(5) The leading partial inverts itself to collapse onto the just-obtained $\gamma$ A Shockley, giving rise to the original stair-rod dislocation $\gamma \delta$ :

$$
\mathrm{A} \delta+\gamma \mathrm{A} \rightarrow \gamma \delta
$$

After this, the leading partial detaches, pulling the trailing partial which completes its reaction with $\delta \alpha$. This enables the resulting $\mathrm{B} \alpha$ partial to slightly glide up on facet $(a)$.

(6) Finally, the trailing partial inverts itself to collapse onto $\gamma \delta$ :

$$
\delta \mathrm{B}+\gamma \delta \rightarrow \gamma \mathrm{B}
$$

after which it detaches. $\gamma \mathbf{B}$ also glides up on $(c)$, leaving a slightly truncated SFT with no base and no possibility of reconstruction. 